\title{
Analysis of non-adiabatic heat-recirculating combustors
}

\author{
Paul D. Ronney \\ Department of Aerospace and Mechanical Engineering \\ University of Southern California \\ Los Angeles, CA 90089-1453 USA
}

Address correspondence to:

Prof. Paul D. Ronney

Department of Aerospace and Mechanical Engineering

University of Southern California

Los Angeles, CA 90089-1453

(213) 740-0490

(213) 740-8071 (fax)

ronney@usc.edu

Full citation: Ronney, P. D., "Analysis of non-adiabatic heat-recirculating combustors," Combustion and Flame, Vol. 135, pp. 421-439 (2003). 


\title{
Analysis of non-adiabatic heat-recirculating combustors
}

\author{
Paul D. Ronney \\ Department of Aerospace and Mechanical Engineering \\ University of Southern California \\ Los Angles, CA 90089-1453 USA
}

\begin{abstract}
A simple first-principles model of counter-current heat-recirculating combustors is developed, including the effects of heat transfer from the product gas stream to the reactant stream, heat loss to ambient and heat conduction in the streamwise direction through the dividing wall (and heat transfer surface) between the reactant and product streams. It is shown that streamwise conduction through wall has a major effect on the operating limits of the combustor, especially at small dimensionless mass fluxes $(M)$ or Reynolds numbers that would be characteristic of microscale devices. In particular, if this conduction is neglected, there is no small- $M$ extinction limit because smaller $M$ leads to larger heat recirculation and longer residence times that overcome heat loss if $M$ is sufficiently small. In contrast, even a small effect of conduction along this surface leads to significantly higher minimum $M$. Comparison is made with an alternative configuration of a flame stabilized at the exit of a tube, where heat recirculation occurs via conduction through tube wall; it is found that the counter-current exchanger configuration provides superior performance under similar operating conditions. Implications for microscale combustion are discussed.
\end{abstract}




\section{Introduction}

Recently interest in heat-recirculating "excess enthalpy” burners, first studied over 30 years ago $[1,2]$, has been renewed due to efforts in microscale combustion and power generation $[3,4,5,6]$. Such work is motivated by the fact that hydrocarbon fuels contain 100 times more energy per unit mass than lithium-ion batteries, thus devices converting of fuel to electricity at better than $1 \%$ efficiency represent improvements in portable electronic devices and other battery-powered equipment. At small scales, however, heat and friction losses become more significant, thus devices based on existing macroscale designs such as internal combustion engines may be impractical. Consequently, many groups have considered heat recirculation using a counter-current heat exchanger for thermal management. By transferring thermal energy from the combustion products to the reactants without mass transfer (thus dilution of reactants), the total reactant enthalpy (sum of thermal and chemical enthalpy) is higher than in the incoming cold reactants and therefore can sustain combustion under conditions (lean mixtures, small heating value fuels, large heat losses, etc.) that would lead to extinguishment of the flame without recirculation.

Apparently there has been only one modeling study of extinction limits and limit mechanisms in heat-recirculating burners. In 1978 Jones et al. [7] performed a global energy balance on the reactant and product streams in heat-recirculating combustors using empirically specified minimum reaction temperatures and prescribed heat losses. Despite its simplicity, two extinction limits were predicted, a blow-off type limit at large dimensionless mass flux $(M)$ or Reynolds number $(R e)$ and another limit at small $M$ due to heat losses. For high- $M$ limit, the minimum fuel concentration supporting combustion increases with increasing $M$ because as $M$ increases, the residence time decreases, thus a higher reaction rate and consequently higher reaction temperature and therefore a higher fuel concentration is required to sustain combustion. For the low- $M$ limit the minimum fuel concentration increases with decreasing $M$ because the heat loss rate was assumed fixed; the extinction limit criterion of a minimum reaction temperature corresponds essentially to a fixed ratio of heat loss rate to heat generation rate, thus as the mass flow rate decreases a higher mass fraction of fuel is needed to produce the minimum required heat generation rate to avoid extinction. These predictions are qualitatively similar to those seen in heat recirculating combustor experiments [1-3]. Moreover, dual-limit behavior (i.e. 
a high-velocity and a low-velocity limit for fixed fuel concentration) is characteristic of many combustion systems [8].

While instructive, the Jones et al. model [7] is not entirely predictive because an empirical quantity is required, specifically the minimum reactor temperature supporting combustion, plus the rate of heat loss (not just a heat loss coefficient) must be prescribed. Perhaps more importantly, we will show that small- $M$ extinction limits (which are most relevant to microscale applications) requires a process besides heat losses, such as heat conduction along the dividing wall between reactant and product streams. Low- $M$ limits were predicted in [7] because heat losses to ambient were prescribed independent of $M$, whereas for realistic heat transfer models, these losses will decrease with decreasing $M$ as discussed later. With realistic heat recirculation and heat loss models, at low $M$ the recirculation and loss track each other, thus no low- $M$ extinction limit is predicted unless an additional is present.

Consequently, this work aims to develop the simplest possible first-principles model (not requiring specification of an empirical minimum reaction temperature or a prescribed heat loss) of heat transfer, finite-rate exothermic chemical reaction, streamwise wall thermal conduction and heat loss in a counter-current heat exchanger/combustor and describe the resulting extinction mechanisms. Comparison is made with an alternative configuration also reported in the literature $[9,10]$, namely that of a flame stabilized at the exit of a tube, where heat recirculation occurs via conduction through tube wall rather than in a counter-current heat exchanger.

\section{Counter-current heat exchanger/combustor}

\section{Approach}

We consider a linear counter-current heat exchanger (Fig. 1) with intake (for premixed reactants) and exhaust (for combustion products) ports at one end $(x=0)$. At the opposite end $(x$ $=L)$ a well-stirred reactor (WSR) is stationed between the reactant (low-temperature) and product (high-temperature) sides of the exchanger. A thermally conductive dividing wall separates the two sides of the exchanger. The current analysis could be extended to a concentric tube counter-current heat exchanger, which has also been considered for both macroscale [1] and microscale [10] heat-recirculating combustors, if separate values of $h_{1}$ are chosen for the outer 
annual reactant stream and the inner tubular product stream and (because of the axis of symmetry) heat loss from the product stream is set to zero. The use of a WSR combustion model is not arbitrary; initial experimental and numerical results [3] show that near extinction limits, reaction zone structures in such burners are very different from propagating premixed flames; instead, there are much larger (compared to propagating flames) reaction zones, smaller temperature gradients, lower peak temperatures, longer residence times at high temperature, and no visible flame emission. These characteristics are typical of "flameless" or "mild" combustion observed in burners employing highly preheated air $[11,12,13]$. The WSR model represents a limit of mild combustion where heat and mass diffusion effects are negligible (in the sense that they are so rapid that temperature and composition gradients within the reactor cannot be sustained) and transport is limited entirely by convection.

With the WSR model, "blow-off" extinction limits occur at large $M$ where residence times in the WSR are insufficient compared to chemical reaction times. We will show that limits also exist at small $M$ in non-adiabatic heat-recirculating burners with wall conduction in the streamwise direction. This is because as $M$ decreases, the fraction of heat release transferred away from the WSR due to conduction along the wall (and subsequently transferred to the gas streams, then lost to ambient) increases. This causes the WSR temperature to decrease, and because reaction time increases exponentially with decreasing temperature, at sufficiently small $M$ the reaction time becomes larger than the residence time - even though residence times and heat recirculation gains in enthalpy both increase as $M$ decreases. Without wall conduction, this low- $M$ extinction limit mechanism is absent. The effect of wall conduction on heat exchanger performance has been studied previously in the context of cryogenic systems [14, 15] and microscale heat exchangers $[16,17]$ but apparently not in the context of non-adiabatic combustors.

Experimental and numerical results for heat-recirculating burners [3] show that near extinction limits, the maximum possible heat recirculation (thus maximum excess enthalpy) is needed to sustain reaction, thus reaction occurs inside the WSR volume as specified here, whereas farther from limits less recirculation is required, thus reaction may occur upstream or downstream of the WSR. Consequently, the model employed here is appropriate near extinction limits, which is the main interest, but may be inappropriate away from limits. The operating regime away from extinction limits, where chemical reaction may occur outside the WSR, could 
be treated as a convective-diffusive system of with reaction in a preheat zone of finite, prescribed length, in a manner similar to that analyzed by Zel'dovich [18]. In extreme cases of sufficiently high fuel concentrations and small $M$, this reaction outside the WSR leads to a "flashback" limit because a flame can propagate upstream from the reaction zone to the reactant inlet without any need for heat recirculation. Such a limit cannot be predicted by the current model since the reaction is presumed to stay within the WSR. While this represents another limitation on the operating conditions of a heat-recirculating burner, it does not affect the extinction limits of primary interest in this work.

The configuration employed here is inherently two-dimensional because gas-phase heat conduction is orthogonal to streamwise convection. A one-dimensional model is obtained by (1) using constant overall heat transfer coefficients $\left(h_{1}\right)$ for heat transfer from reactant and product streams to the dividing wall, (2) using constant overall heat loss coefficients $\left(h_{2}\right)$ for heat losses from these streams to ambient and (3) modeling the wall as "thermally-thin." Constant heat transfer coefficients are not only analytically convenient but realistic for laminar flows in plane channels, where the Nusselt number based on hydraulic diameter $\equiv h_{1} d_{H} / k_{g} \approx 7.5$ [19], and thus for channels much wider in the spanwise direction than their height $d, d_{H} \approx 2 d$ and consequently $h_{1} \approx 3.7 \mathrm{~kg} / d$. For strongly turbulent flows roughly $h \sim \dot{m}^{0.8}$ but for laboratory-scale apparatuses with $d$ on the order of a few $\mathrm{mm}$ and gas velocities of a few hundred $\mathrm{cm} / \mathrm{s}$ at most, Reynolds numbers will be at most a few hundred and thus turbulent flow is not expected. Of course, for microscale devices laminar flow will be ubiquitous. The thermally-thin assumption, which is common in modeling of flame spread over thin solid fuel beds [20], requires that the wall thermal resistance $\square k_{w}$, where $\square$ is the wall thickness and $k_{w}$ its thermal conductivity, is small compared to the channel thermal resistance $1 / h_{1}$ so that temperature drops across the wall are negligible. Since $N u \approx 3.7, h_{1} \approx 3.7 k_{g} / d$, the thermally-thin assumption requires $0.52 k_{w} d / k_{g} \square>>$ 1. For realistic materials $k_{w}>>k_{g}$ and for most practical burners and proposed microscale devices $d / \square \geq 1$, thus this assumption is justified. With the thermally-thin model $T_{w, e}-T_{w, i}<<$ $T_{e}-T_{i}$, where $T_{i}(x)$ is the mean reactant-side gas temperature, $T_{w, i}(x)$ the corresponding temperature on the wall surface, and $T_{e}(x)$ and $T_{w, e}(x)$ the corresponding product-side temperatures (Fig. 1). Moreover, only streamwise wall conduction needs to be calculated, using the mean wall temperature $T_{w} \approx\left(T_{w, e}+T_{w, i}\right) / 2$. 
For simplicity flow channel entrance effects on $h_{1}$ are neglected. For laminar flow in straight channels the ratio of entrance length $L_{e}$ to $d_{H}$ is approximately $0.04 U d_{H} / \square$. This can be rearranged to read $L_{e} / L \approx 0.60 \mathrm{M} / \mathrm{Pr}$, where $L$ is the length of the exchanger and $M$ is the dimensionless mass flux defined later, and thus for $\operatorname{Pr}=0.7, L_{e} / L \approx 0.85 \mathrm{M}$. For the representative conditions analyzed in this work, the maximum value of $M$ of interest is about 0.2 thus at most only the first $17 \%$ of the exchanger length is influenced by entrance effects (this of course applies to both the low-temperature and high-temperature arms of the exchanger). In the entrance regions $h_{l}$ will be higher than its value for fully-developed flow and thus the overally performance of the combustor will be slightly higher than that estimated based on fully developed flow. For the low- $M$ cases of most interest in this study, entrance effects are negligible.

Many heat-recirculating combustors employ multi-turn spiral exchangers (rather than linear exchangers as analyzed here) for which heat losses in the direction shown in Fig. 1 are minimal, but heat losses in the third dimension still exist. These losses are estimated (for laminar flows) by $H \equiv h_{2} / h_{1}=d / w$, where $w$ is the channel depth in the third dimension. Insulation would not change this estimate substantially because insulating materials have thermal conductivities no lower than air and the insulation thickness in most devices is much less than $w$. Furthermore it is assumed, as in [7], that the WSR volume is small compared to the heat exchanger, thus heat loss from the WSR is neglected.

\section{Analysis}

Heat transfer is divided into three zones: the dividing wall, the reactants side of the heat exchanger and the products side of the heat exchanger. Energy balances on these three zones readily yield (see Nomenclature):

$$
\begin{aligned}
& k_{w} \square \frac{d^{2} T_{w}}{d x^{2}} \square 2 h_{1} T_{w}+h_{1}\left(T_{w, i}+T_{w, e}\right)=0 \\
& \dot{m} C_{P} \frac{d T_{i}}{d x} \square h_{1}\left(T_{w, i} \square T_{i}\right)+h_{2}\left(T_{i} \square T\right)=0
\end{aligned}
$$




$$
\dot{m} C_{P} \frac{d T_{e}}{d x} \square h_{1}\left(T_{e} \square T_{w, e}\right) \square h_{2}\left(T_{e} \square T\right)=0
$$

Using the mean wall temperature $T_{w} \approx\left(T_{w, e}+T_{w, i}\right) / 2$, invoking the thermally-thin assumption $T_{w, e}-T_{i, w}<<T_{e}-T_{i}$, and taking the sum and difference of (2a) and (3a) yield

$$
\begin{aligned}
& \frac{k_{w} \square}{h_{1}} \frac{d^{2} T_{w}}{d x^{2}} \square 2 T_{w}=\square\left(T_{i}+T_{e}\right) \\
& \dot{m} C_{P} \frac{d}{d x}\left(T_{i} \square T_{e}\right)+\left(h_{1}+h_{2}\right)\left(T_{i}+T_{e}\right) \square 2 h_{2} T \square 2 h_{1} T_{w}=0 \\
& \dot{m} C_{P} \frac{d}{d x}\left(T_{i}+T_{e}\right)+\left(h_{1}+h_{2}\right)\left(T_{i} \square T_{e}\right)=0
\end{aligned}
$$

The previous model by Jones et al. [7] is similar to Eqs. (2b) and (3b) applied globally rather than on an element of the heat exchanger of infinitesimal length.

Combining (1b), (2b) and (3b) yields

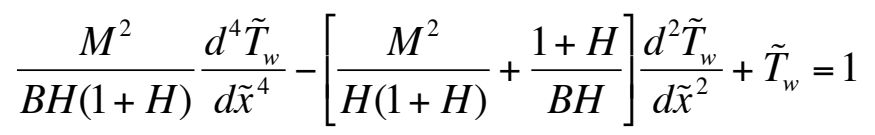

where

$$
M \equiv \frac{\dot{m} C_{P}}{h_{1} L} ; B \equiv \frac{2 h_{1} L^{2}}{k_{w} \square} ; H \equiv \frac{h_{2}}{h_{1}} ; \tilde{x} \equiv \frac{x}{L} ; \tilde{T} \equiv \frac{T}{T}
$$

Thus, the heat transfer problem has three dimensionless parameters, namely the Biot number $(B)$ (scaled by $L / \square$ since convection occurs along the length of the dividing wall $(L)$ whereas conduction along the wall occurs through a cross-section of height $\square$, a heat loss coefficient $(H)$ and the mass flux $(M)$. Since the heat transfer coefficient $h_{1}$ is assumed to be the same on both sides of the dividing wall and the dividing wall is assumed to be thermally thin (negligible thermal resistance), the overall heat transfer coefficient for the heat exchanger is $h_{1} / 2$, and since $\dot{m} C_{P}$ is the same on both sides of the exchanger, $2 M=\dot{m} C_{P} /\left(h_{1} / 2\right) L$ is equivalent to the Number 
of Transfer Units (NTU) in the heat exchanger literature. Additionally, 2/MB is equivalent to the streamwise wall conduction parameter employed by Kroeger [14] and others.

For laminar flow where $h_{1} \approx 3.7 k_{g} / d, M=\square_{g} U d C_{P} /\left(3.7 k_{g} / \mathrm{d}\right) L=0.26(d / L) R e P r$, where $R e$ $\equiv \square_{g} U d / \square_{g}$ is the Reynolds number and $\mathrm{Pr}$ is the Prandtl number. For geometrically similar burners $(d / L=$ constant), small $M$ corresponds to small $R e$ (characteristic of most microscale devices), thus describing the small- $M$ extinction mechanism is the primary focus of this work.

The solution to (4) is

$$
\tilde{T}_{w}(\tilde{x})=1+c_{1} e^{a \tilde{x}}+c_{2} e^{\square a \tilde{x}}+c_{3} e^{b \tilde{x}}+c_{4} e^{\square b \tilde{x}}
$$

where $c_{1}-c_{4}$ are unknown constants, $\mathrm{a}=\square_{1}^{1 / 2}, \mathrm{~b}=\square_{2}^{1 / 2}$ and $\square_{1}$ and $\square_{2}$ are the roots of

$$
\frac{M^{2}}{B H(1+H)} \square \frac{M^{2}}{=H(1+H)}+\frac{1+H}{B H} \square+1=0
$$

Two boundary conditions are obtained by assuming the dividing wall is adiabatic (thus has zero temperature gradient) at both ends. This is reasonable since the wall cross-sectional areas are generally small compared to channel cross-sectional areas, plus $k_{w}>>k_{g}$, thus there would be little heat transfer out the bare wall ends compared to convective transfer to/from the wall surfaces. With this assumption wall conduction is not a heat loss mechanism, instead it only redistributes thermal energy within the device; it will be shown this still results in a major impact on burner performance. The effect of the wall end boundary conditions is discussed in a later section. From (5) this yields

$$
\begin{aligned}
& a c_{1} \square a c_{2}+b c_{3} \square b c_{4}=0 \\
& a c_{1} e^{a} \square a c_{2} e^{\square a}+b c_{3} e^{b} \square b c_{4} e^{\square b}=0
\end{aligned}
$$

Two boundary conditions are obtained by substituting (5) into (1b) and applying $\tilde{T}_{i}(0)=1$ (ambient inlet temperature) yielding 


$$
\begin{aligned}
& \frac{B a^{2}}{\square B} \square 2 \mathrm{E}_{1}+\frac{B a^{2}}{\square B} \square 2 \mathrm{~B}_{c_{2}}+\frac{\mathrm{B} b^{2}}{\square B} \square 2 \mathrm{C}^{3}+\frac{\mathrm{B} b^{2}}{\square B} \square 2 \mathrm{~B}_{c_{4}}=\square \tilde{T}_{e}(0)+1
\end{aligned}
$$

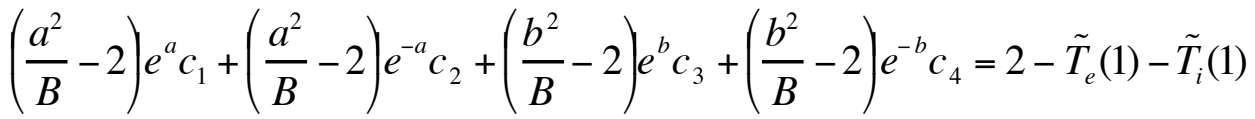

where the exhaust temperature $\tilde{T}_{e}(0)$ and WSR inlet and outlet temperatures $\tilde{T}_{i}(1)$ and $\tilde{T}_{e}(1)$ are all unknowns.

Two additional equations are obtained by manipulating (2b) and (3b) to isolate $\tilde{T}_{i}(\tilde{x})$ and $\tilde{T}_{e}(\tilde{x})$ as functions of $\tilde{T}_{w}(\tilde{x})$ only and applying the boundary conditions at $\tilde{x}=0$ and $\tilde{x}=1$. In non-dimensional form the equations for $\tilde{T}_{i}(\tilde{x})$ and $\tilde{T}_{e}(\tilde{x})$ as functions of $\tilde{T}_{w}(\tilde{x})$ only are

$$
\begin{aligned}
& M \frac{d \tilde{T}_{i}}{d \tilde{x}}+(1+H) \tilde{T}_{i} \square H=\tilde{T}_{w} \\
& M \frac{d \tilde{T}_{e}}{d \tilde{x}} \square(1+H) \tilde{T}_{e}+H=\square \tilde{T}_{w}
\end{aligned}
$$

Substituting in $\tilde{T}_{w}(\tilde{x})$ from Eq. (5) and applying the boundary condition $\tilde{T}_{i}(0)=1$ to $(8)$, the solutions to (8) and (9) are

$$
\begin{aligned}
\tilde{T}_{i}(\tilde{x})= & \square\left(d_{1}+d_{2}+d_{3}+d_{4}\right) e^{\square(1+H) \tilde{x} / M}+d_{1} e^{a \tilde{x}}+d_{2} e^{\square a \tilde{x}}+d_{3} e^{b \tilde{x}}+d_{4} e^{\square b \tilde{x}}+1 \\
\tilde{T}_{e}(\tilde{x})= & \left(\tilde{T}_{e}(0) \square\left(1+f_{1}+f_{2}+f_{3}+f_{4}\right)\right) e^{(1+H) \tilde{x} / M} \\
& +1+f_{1} e^{a \tilde{x}}+f_{2} e^{\square a \tilde{x}}+f_{3} e^{b \tilde{x}}+f_{4} e^{\square b \tilde{x}}
\end{aligned}
$$

where

$$
\begin{aligned}
& d_{1}=\frac{c_{1}}{a M+(1+H)} ; d_{2}=\frac{c_{2}}{\square a M+(1+H)} ; d_{3}=\frac{c_{3}}{b M+(1+H)} ; d_{4}=\frac{c_{4}}{\square b M+(1+H)} \\
& f_{1}=\frac{c_{1}}{\square a M+(1+H)} ; f_{2}=\frac{c_{2}}{a M+(1+H)} ; f_{3}=\frac{c_{3}}{\square b M+(1+H)} ; f_{4}=\frac{c_{4}}{b M+(1+H)} .
\end{aligned}
$$

Equations (10) and (11) can then be applied at $\tilde{x}=1$ to obtain 


$$
\begin{aligned}
& \tilde{T}_{i}(1)=\square\left(d_{1}+d_{2}+d_{3}+d_{4}\right) e^{\square(1+H) / M}+d_{1} e^{a}+d_{2} e^{\square a}+d_{3} e^{b}+d_{4} e^{\square b}+1 \\
& \tilde{T}_{e}(1)=\left(\tilde{T}_{e}(0) \square\left(1+f_{1}+f_{2}+f_{3}+f_{4}\right)\right) e^{\square(1+H) / M}+f_{1} e^{a}+f_{2} e^{\square a}+f_{3} e^{b}+f_{4} e^{\square b}+1
\end{aligned}
$$

where again $\tilde{T}_{e}(0), \tilde{T}_{i}(1)$ and $\tilde{T}_{e}(1)$ are all unknowns.

The final relationship needed to close the system of equations is obtained from the WSR model [21]. For the simplest case of first-order single-step Arrhenius chemical reaction, the relationship between WSR inlet temperature $T_{i}(1)$, outlet temperature $T_{e}(1)$ and mass flux is

$$
\frac{\dot{m}}{\square_{g} Z A_{R}}=\frac{\left(T_{i}(1)+\square T\right) \square T_{e}(1)}{T_{e}(1) \square T_{i}(1)} \exp \frac{E}{R T_{e}(1)} \stackrel{\square}{\square}
$$

where the term $T_{i}(1)+\square T$ is the adiabatic flame temperature based on an inlet temperature (to the WSR) of $T_{i}(1)$. In dimensionless form Eq. (14) becomes

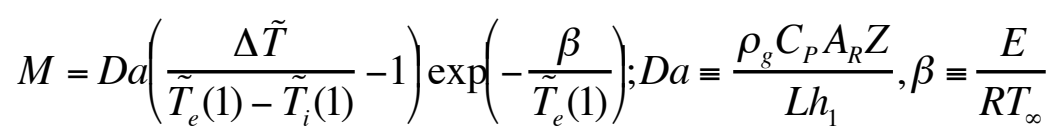

where $D a$ is the Damköhler number and $\square$ the Zeldovich number. For fixed $\tilde{T}_{i}(1)$, the response of $\tilde{T}_{e}(1)$ to $M$ produces well-known Z-shaped curves, however, for the current problem $\tilde{T}_{e}(1)$ is strongly affected by $M$ due to combined effects of heat recirculation, heat loss and wall conduction, thus, the relationships between $M$ and $\tilde{T}_{e}(1)$ take many forms.

Equations $(7 \mathrm{a}-7 \mathrm{~d}, 12,13$ and 15) represent 6 linear and one non-linear equation for seven unknowns $\mathrm{c}_{1}-\mathrm{c}_{4}, \tilde{T}_{e}(0), \tilde{T}_{i}(1)$ and $\tilde{T}_{e}(1)$. These are readily solved for various values of the dimensionless parameters that completely define the problem, namely the heat transfer parameters $M, B$ and $H$ and the combustion parameters $\sqcap \tilde{T}, D a$ and $\square$. 


\section{Choice of baseline numerical parameters}

Baseline dimensionless parameters that semi-quantitatively represent macroscale heatrecirculating burner experiments should be chosen. $H=0.05$ is employed, corresponding to $d=$ $3.9 \mathrm{~mm}$ and $w=78 \mathrm{~mm}$ as in our initial experiments $[3,22]$. A Zel'dovich number $(\square)$ of 70 is chosen, corresponding to $E=42 \mathrm{kcal} /$ mole (typical of hydrocarbon oxidation [21]). For experiments [3] using 3-turn Inconel burners having $d \approx 3.9 \mathrm{~mm}, \square \approx 0.51 \mathrm{~mm}, L \approx 580 \mathrm{~mm}, k_{w} \approx$ $11.4 \mathrm{~W} / \mathrm{mK}$, thus $B \approx 2900$, an extinction limit occurs at $\sqcap \tilde{T} \approx 1.5$ for $M=0.2 ; D a \approx 10^{7}$ leads to extinction at these conditions and is employed in the calculations below.

\section{Results - infinite reaction rates}

First, heat transfer characteristics of counter-current heat exchangers are examined for infinite reaction rate $(D a \square \infty)$, thus $\tilde{T}_{e}(1)-\tilde{T}_{i}(1)=\sqcap \tilde{T}$. Figure 2 (top) shows temperature profiles for adiabatic $(H=0)$ and wall-conduction-free $(B \square \infty)$ conditions, for which profiles are linear. For all $M$, the exhaust temperature $\tilde{T}_{e}(0)$ is the adiabatic flame temperature $1+\sqcap \tilde{T}$. The

WSR temperature $\tilde{T}_{e}(1)$ increases monotonically as $M$ decreases (Fig. 3) because as $M$ decreases the heat transfer rate $h_{l} L \sqcap \tilde{T}$ is constant but $\dot{m}$ decreases, thus the heat transfer per unit mass increases. It can be shown that

$$
\tilde{T}_{e}(1)=1+\square \tilde{T} \square+\frac{1}{2 M} \square(D a \square \quad, H=0, B \square \quad)
$$

which is equivalent to the well-known result from the heat exchanger literature $\square=$ $1 /\left(1+(\mathrm{NTU})^{-1}\right)$, where $\square$ is the effectiveness, for exchangers with no streamwise wall heat conduction and equal $\dot{m} C_{P}$ for the two streams. Note that for $M \square \infty$, there is no heat recirculation, thus the reactor temperature $\tilde{T}_{e}(1)$ approaches the adiabatic flame temperature for cold reactants $=1+\sqcap \tilde{T}$.

With substantial heat loss (Fig. 2, middle) the profiles are quite different; temperatures are ambient except near the WSR $(\tilde{x}=1)$. The WSR temperature $\tilde{T}_{e}(1)$ is lower than for $H=0$ 
but because $D a \square \infty$ was assumed, the combustion-induced temperature rise $\tilde{T}_{e}(1)-\tilde{T}_{i}(1)$ is still $\sqcap \tilde{T}$. This case should still be considered an excess enthalpy burner because reaction temperatures are higher than adiabatic flame temperatures without recirculation, i.e., $\tilde{T}_{e}(1)>$ $1+\sqcap \tilde{T}$. Figure 3 shows that $\tilde{T}_{e}(1)$ asymptotes to a fixed value as $M \square 0$ and does not decrease to ambient because as $M \square 0$, heat recirculation is balanced by heat loss; below a certain value of $M$, decreasing $M$ further (e.g. in an experiment by decreasing the mass flow rate or increasing the length of the heat exchanger) has no effect other than to increase the fraction of the length of the exchanger where both the reactant and product streams remain at near-ambient temperatures. It can be shown that the reactor temperature in the limit $M \square 0$ is

$$
\tilde{T}_{e}(1)=\frac{(1+\square \tilde{T}) \square \square 1}{\square \square 1} ; \square \equiv \sqrt{\frac{1+2 H+\sqrt{4 H(1+H)}}{1+2 H \square \sqrt{4 H(1+H)}}}(D a \square \quad, B \square \quad, M \square 0)
$$

This observation is extremely important to understanding the low- $M$ extinction limits because it indicates that, even in the presence of heat losses, without wall conduction there is no means to reduce the reactor temperature as $M$ is decreased. This is quite different from combustors without heat recirculation, where sufficient reduction in mass flow rate will nearly always lead to extinction due to heat losses.

With substantial wall heat transfer $(B \neq 0)$ but adiabatic conditions $(H=0)$ (Fig. 2, bottom), the temperature profiles clearly show that wall conduction removes thermal energy from the high-temperature gas near $\tilde{x}=1$ and returns energy to the gas at lower temperatures (smaller $\tilde{x}$ ). Figure 3 shows that at small $M$ wall conduction dominates and the WSR temperature $\tilde{T}_{e}(1)$ is far below that for $B \square \infty$ (even though the system is still adiabatic) whereas for large $M$ wall heat transport is insignificant because gas-phase convection dominates wall conduction. The exit temperature $\tilde{T}_{e}(0)$ is still $1+\sqcap \tilde{T}$ because the system is adiabatic. In the limit $M \square 0, \tilde{T}_{e}(\tilde{x}), \tilde{T}_{i}(\tilde{x})$ and $\tilde{T}_{w}(\tilde{x})$ (not shown) all take on the value of $1+\sqcap \tilde{T}$ except very near $\tilde{x}=0$, where $\tilde{T}_{i}(\tilde{x}) \square 1$, and near $\tilde{x}=1$, where $\tilde{T}_{e}(1) \square 1+2 \sqcap \tilde{T} \quad(=4$ for the numerical values employed in Fig. 3) due to heat recirculation caused by thermal conduction along the wall. 


\section{Results - finite-rate chemistry}

Figure 4 shows the effect of mass flux on WSR temperature for $D a=10^{7}$. Without heat recirculation the $H=0$ curve would have the Z-shape typical of WSRs; with heat recirculation, at small $M$ (thus large $\tilde{T}_{e}(1)$ and fast reaction) the upper branch follows the $D a \square \infty$ limit (Eq. (16)). The lower branch is probably unstable as in conventional WSRs. (For plotting clarity the lowest, extinguished branch where $\tilde{T}_{e}(1) \approx 1$ is not shown.)

For no wall conduction $(B \square \infty)$, there are no solutions for sufficiently large $M$ and two solutions for all smaller $M$, even for non-adiabatic conditions. Thus, without wall conduction combustion is possible at arbitrarily small $M$ (or Re). In fact, the highest $\tilde{T}_{e}(1)$ (thus highest reaction rates and longest residence times, farthest from extinction) occur at the smallest $M$. This could be expected based on small-M behavior for $D a \square \infty$ (Fig. 3). Experiments [1-3] show that small- $M$ limits do indeed exist, indicating that an additional factor is required to model small- $M$ extinction limits.

In contrast, with wall conduction (finite B), the response curves become isolas with both lower and upper limits on M because conduction of thermal energy away from the WSR vicinity through the wall becomes significant at small $M$. Once conducted away from the WSR vicinity, some thermal energy is transferred back to the gas via convection and a portion of this energy is then lost to ambient. It is emphasized that this mechanism is important only at small $M$, where wall conduction is competitive with gas-phase convection. Figure 4 also shows that the large- $M$ extinction limit is extended slightly by wall conduction, since heat recirculation (thus WSR temperature) is low at large $M$ (Eq. 16), thus the increase in heat recirculation provided by wall conduction increases the WSR temperature slightly. This behavior is discussed in more detail in the section on the conductive-tube burner analysis.

For non-adiabatic small- $M$ cases, temperature profiles are similar to Fig. 2 (middle) in which all heat generated is lost to ambient before the products reach the burner exit. Thus, simply equating rates of heat generation and loss does not yield extinction criteria because all heat generated can be lost to ambient rather than exhausted at the exchanger exit without extinction occurring. Jones et al. [7] predicted low- $M$ extinction limits because fixed heat loss was assumed; at low M heat loss would exceed heat generation and extinction occurs. In contrast, heat loss is calculated systematically in this work. 
Figure 5 shows the effect of $B$ and thus wall conduction on the reactor temperature $\tilde{T}_{e}(1)$ for several fixed values of $M$. The general C-shape of these curves is the same for all values of $M$ but the value of $B$ at the turning point is a non-monotonic function of $M$. The most robust value of $M$, corresponding to the lowest value of $B$ at the turning point, is about 0.1 as would be expected from the isolas seen in Fig. 4. As was seen in Fig. 3, the maximum (and minimum, on the lower branch of solutions) values of $\tilde{T}_{e}(1)$ occur at the lowest $M$. As $M$ increases, the curve flattens until it disappears entirely at $M \approx 0.3$.

Figure 6 shows the effect of $M$ on the minimum $\sqcap \tilde{T}$ supporting combustion (corresponding to the minimum fuel concentration, thus extinction limit). As in Fig. 4, without wall conduction $(B \square \infty)$ no small- $M$ extinction limit exists. For finite $B$, both small- $M$ and large$M$ limits exist. Consistent with Fig. 4, the large- $M$ limit is slightly extended by decreasing $B$ whereas the small- $M$ limit is drastically narrowed by decreasing $B$.

It should be stressed that the value of $B$ needed to affect extinction is much smaller than that expected based on simplistic estimates. The overall ratio of streamwise convection to wall conduction is $\dot{m} C_{P} /\left(k_{w} \square L L\right)=M B / 2$. For the $B=10,000$ curve in Fig. 6, extinction limits are affected for all $M \leq 0.01$, thus $M B / 2 \leq 50$. Based on simplistic estimates, no effect wall conduction effects would be expected unless $M B / 2 \leq 1$. The powerful wall conduction effects result from the large wall temperature gradients near the WSR when heat losses are present (Fig. 2, middle), which are much larger than the mean gradient under these conditions.

In Fig. 6, $M$ is plotted on a logarithmic scale for clarity. When plotted linearly the curve is very steep on the small- $M$ extinction branch but is much shallower on the large- $M$ branch. This trend is very similar to that seen in experiments [1-3].

Figure 7 shows the effect of $M$ on the minimum value of $B$ supporting combustion for $\sqcap \tilde{T}=1.5$. The same trends as demonstrated above can be seen: both small- $M$ and large- $M$ extinction limits exist for non-adiabatic conditions, the small- $M$ limits are much narrower at small $B$ (and disappear as $B \square \infty$ ) and large- $M$ limits are extended slightly by wall conduction. Also, for the special case $H=0, B \approx 9$, as $M$ is decreased conditions change from extinguished to burning to extinguished and finally back to burning again. This odd behavior results from interactions of increasing conduction-free $\tilde{T}_{e}(1)$ (Eq. 16), increasing wall conduction effects and increasing residence time as $M$ decreases. In particular, as $M$ decreases, the drop in temperature leads to a small- $M$ extinction limit, even for adiabatic cases, if the decrease in $M$ decreases the 
right side of Eq. (15) (i.e., the reaction rate drops due to a drop in the reaction temperature $\tilde{T}_{e}(1)$ ) more than the decrease in $M$ increases the reactor temperature. Of course, since the minimum $\tilde{T}_{e}(1)$ for adiabatic conditions is $1+2 \sqcap \tilde{T}$, at sufficiently small $M$, the extinction limit will always disappear for adiabatic conditions, meaning that there could be multiple extinction limits changing only $M$. Figure 7 (and additional calculations not shown) reveal that this behavior was found only for narrow ranges of $B$ and probably would not be experimentally observable.

\section{Effect of boundary conditions}

To test the effects of the assumption (Eqs. 7a and 7b) that the ends the dividing wall are adiabatic, the opposite case of convection boundary conditions on the ends was examined. At the inlet end of the exchanger, the most natural choice would probably be to assume a heat loss per unit area $h_{2}\left(T_{w}(0)-T_{\infty}\right)$ across the entire thickness $\square$ of the wall that balances the conductive flux $k_{w}\left(\mathrm{~d} T_{w} / \mathrm{d} x\right)_{\mathrm{x}=0}$. This can be written as

$$
\left(a \square H B^{*}\right) c_{1}+\left(\square a \square H B^{*}\right) c_{2}+\left(b \square H B^{*}\right) c_{3}+\left(\square b \square H B^{*}\right) c_{4}=0 ; B^{*} \equiv B \frac{\square}{2 L}=\frac{h_{1} L}{k_{w}}
$$

where the additional geometrical parameter $\square 2 L$ that did not appear as a separate parameter in the analysis for adiabatic wall ends must now be specified. Substitution of Eq. (7a') for (7a) was found to have negligible effect on the results except for the unrealistic case of $\square 2 L$ near or larger than unity. This is because at low $M$, in the region near $\tilde{x}=0, \tilde{T}_{w}(0)$ is near ambient temperature because of heat loss at larger $\tilde{x}$ (See Fig. 2b) and thus no additional heat loss results from the change in boundary condition, whereas at high $M$, heat losses are inconsequential even over the much larger surface area of the exchanger itself. For the WSR end of the exchanger, the most natural choice would probably be to assume a heat transfer per unit area $h_{1}\left(\bar{T}(1)-T_{w}(1)\right)$, where $\bar{T}(1)=\left(T_{i}(1)+T_{e}(1)\right) / 2$ is the mean gas temperature seen by the wall end (see Fig. 1), across the entire thickness $\square$ of the wall that balances the conductive flux $k_{w}\left(\mathrm{~d} T_{w} / \mathrm{d} x\right) \tilde{x}=1$. This boundary condition can be written as 


$$
\left(a+B^{*}\right) e^{a} c_{1}+\left(\square a+B^{*}\right) e^{\square a} c_{2}+\left(b+B^{*}\right) e^{b} c_{3}+\left(\square b+B^{*}\right) e^{\square b} c_{4}=B^{*} \underset{\tilde{T}_{i}(1)+\tilde{T}_{e}(1)}{2} \square 1=\left(7 \mathrm{~b}^{\prime}\right)
$$

Figure 8 shows a comparison of predictions obtained using Eqs. (7a) and (7b) to those obtained with (7a') and (7b'). It would be misleading to show results as a function of the new geometrical parameter $\square 2 L$ for fixed $B$ and $M$ since the only way to vary $\square 2 L$ for fixed $B$ and $M$ would be by varying the wall thermal conductivity $k_{w}$ according to $k_{w} \sim \square^{1}$, and varying $k_{w}$ alone would have a drastic effect burner performance. Consequently, results are shown for varying $\square$ with all other dimensional parameters fixed, meaning that as $\square 2 L$ is increased, $B$ decreases proportionally so that $B^{*}$ is fixed. It can be seen in Fig. 8a that for $B^{*}=1$, which is representative of our initial experiments $[3,22]$, there is practically no difference between the adiabatic and convection boundary conditions, although another branch of solutions appears at very low $B$. This branch is considered non-physical because, as Fig. 8b shows, it corresponds to large values of $\square 2 L$ (i.e., a heat exchanger that is nearly as large in the spanwise direction as it is in the streamwise direction). The current model does not include heat losses from the WSR end of the burner that would become critical for this aspect ratio. The high- $B$ branch of solutions shown in Fig. 8a was found to be almost independent of $B^{*}$ except for large values of $\square 2 L$. These results indicate that the choice of adiabatic wall ends is reasonable for the conditions where the current model is asserted to be valid.

\section{Combustors with conductive tube walls}

\section{Analysis}

Experiments [9] have shown that by holding combustion on the end of a thermallyconductive tube through which the reactants pass and are preheated by the tube wall, combustion can be sustained under conditions (specifically tubes whose diameters are smaller than the quenching distance) that could not sustain combustion without heat recirculation. This conductive-tube configuration provides an alternative to the counter-current or Swiss-roll heat exchanger for recycling a portion of the heat release back to the reactants. Thus, it is of interest 
to compare the performance of counter-current and conducting-tube heat-recirculating combustors.

The configuration of Fig. 1 (lower) is employed in order to make the comparison of the two types of heat recirculating combustors as valid as possible. Heat transfer is divided into two zones: the tube or channel wall and the gas. The thermally-thin wall transports heat along its length, transfers heat to/from the reactant stream with heat transfer coefficient $h_{1}$, and loses heat to ambient with coefficient $h_{2}$. In addition to streamwise convection, heat transport in the gas is via convection to the wall. A no-flux condition is imposed at the line of symmetry in the gas. The configuration shown could be either a cylindrical tube or plane channel, the only difference being the choice of Nusselt number for computing $h_{1}$ (3.7 for the plane channel vs. 4.4 for the tube [19]).

As with the counter-current configuration, ambient gas temperature and adiabatic wall conditions are assumed at $x=0$. The adiabatic wall boundary condition cannot be applied at the WSR end of the tube, otherwise there would be no heat transfer from the combustion zone to the tube and thus no gas preheating would occur. In general the boundary condition will depend on the details of way in which the reaction zone is anchored to the end of the tube and would involve additional length scales and/or heat transfer coefficients. To avoid needing to specify additional parameters, it is assumed that the WSR combustion is still described by Eq. (15) and is followed by a short zone (short enough compared to the tube length $L$ that heat losses can be neglected) downstream of the WSR where heat is transferred to the wall (assumed isothermal through the WSR and this downstream zone) until the gas and wall temperatures are equal. The thermal energy transferred from the gas to the wall is then conducted along the wall in the upstream direction. Clearly this boundary condition represents an upper bound on the performance of the conducting-tube combustor since (1) the heat transfer from the burnt gas to the tube wall does not diminish the WSR temperature, which would reduce the reaction rate and (2) the maximum possible energy is transferred from the burnt gas to the tube wall and subsequently recycled back to the gas upstream of the WSR. In practice this boundary condition would require a heat transfer coefficient in this downstream thermal equilibration zone much higher than $h_{1}$ since this zone is much shorter than $\mathrm{L}$ and thus has much less area available for heat transfer to the gas. (Alternatively this zone could have a much lower loss coefficient $h_{2}$.) 
Energy balances on the wall and the gas readily yield, using the same nondimensionalization as for the counter-current heat exchanger analysis,

$$
\begin{aligned}
& \frac{2}{B} \frac{d^{2} \tilde{T}_{w}}{d \tilde{x}^{2}} \square(1+H) \tilde{T}_{w}=\square\left(\tilde{T}_{i}+H\right) \\
& M \frac{d \tilde{T}_{i}}{d \tilde{x}}+\tilde{T}_{i}=\tilde{T}_{w}
\end{aligned}
$$

which can be combined to obtain

$$
\frac{d^{3} \tilde{T}_{w}}{d \tilde{x}^{3}}+\frac{1}{M} \frac{d^{2} \tilde{T}_{w}}{d \tilde{x}^{2}} \square \frac{B(1+H)}{2} \frac{d \tilde{T}_{w}}{d \tilde{x}} \square \frac{B H}{2 M} \tilde{T}_{w}=\square \frac{B H}{2 M}
$$

The solution to (20) is

$$
\tilde{T}_{w}(\tilde{x})=1+c_{1} e^{a \tilde{x}}+c_{2} e^{b \tilde{x}}+c_{3} e^{d \tilde{x}}
$$

where $c_{1}-c_{3}$ are unknown constants and $\mathrm{a}, \mathrm{b}$ and $\mathrm{g}$ are the roots of

$$
\left\lceil+\frac{1}{M} \square \square \frac{B(1+H)}{2} \square \square \frac{B H}{2 M}=0\right.
$$

One boundary condition is obtained by assuming the tube wall is adiabatic (thus has zero temperature gradient) at the inlet end, thus

$$
a c_{1}+b c_{2}+g c_{3}=0
$$

In dimensional terms, the energy balance corresponding to the aforementioned wall boundary condition at $x=L$ is

$$
\left.\square k \square \frac{d T_{w}}{d x}\right|_{x=L}=\dot{m} C_{P}\left(T_{w}(L) \square T_{e}(L)\right)
$$


where for consistency with the counter-current exchanger analysis the notation $T_{\mathrm{e}}(\mathrm{L})$ is used to denote the dimensional WSR temperature. By applying Eq. (21) at $\tilde{x}=1$, Eq. (24a) can be written as

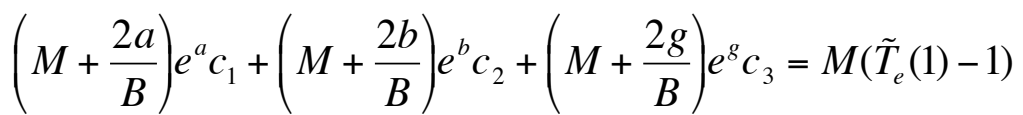

where the dimensionless WSR temperature $\tilde{T}_{e}(1)$ is unknown.

Two additional relations are obtained by substituting (23) into (18) to obtain an expression for $\tilde{T}_{i}(\tilde{x})$ and applying this expression at $\tilde{x}=0$ and $\tilde{x}=1$ to yield, respectively,

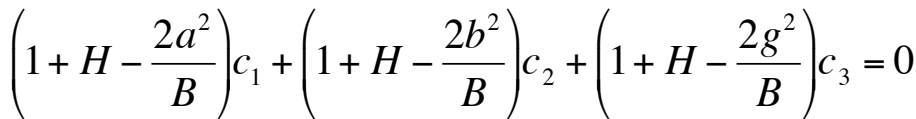

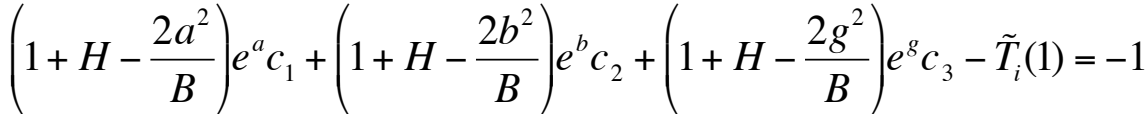

where the condition $\tilde{T}_{i}(0)=1$ has been used in $(25)$ and $\tilde{T}_{i}(1)$ is unknown.

Results

Equations (15), (23), (24b), (25) and (26) represent 1 nonlinear equation and 4 linear equations for the unknowns $c_{1}-c_{3}, \tilde{T}_{i}(1)$ and $\tilde{T}_{e}(1)$. In order to make the comparison between the counter-current and conducting-tube heat-recirculating combustors as valid as possible, the same representative reaction rate parameters $D a=10^{7}$ and $\square=70$ and heat loss parameter $H=$ 0.05 are employed in this section. Nevertheless, the previous baseline heat release parameter $\sqcap \tilde{T}=1.5$ could not be employed because with these reaction rate, heat loss and heat release parameters, there were no solutions to the governing equations for any value of Biot number $B$ except at extremely low values of mass flux $M\left(<10^{-3}\right)$. Consequently, the baseline heat release 
parameter was increased $50 \%$ to $\sqcap \tilde{T}=2.25$; at this value of $\sqcap \tilde{T}$ the maximum values of $M$ and $\tilde{T}_{e}(1)$ were similar for the two types of combustors.

Figure 9 shows the effect of mass flux $M$ on WSR temperature for the conductive-tube configuration (analogous to Fig. 4 for the counter-current configuration). As with the countercurrent configuration, with heat loss these plots are isolas indicating maximum and minimum values of $M$ supporting combustion. Unlike the counter-current combustor, however, decreasing $B$ substantially increases the maximum $M$ because in the conductive-tube case heat conduction along the wall (whose effect is proportional to $1 / B$ ) is the only means to accomplish heat recirculation. Significantly, however, is that the isolas for different values of $B$ are nearly concentric in the counter-current case but are displaced to lower $M$ as $B$ increases for the conductive-tube case. This indicates that for both counter-current and conductive-tube cases, increases wall heat conduction (i.e. lower $B$ ) raises the minimum value of $M$ supporting combustion. Figure 10 illustrates why wall heat conduction, which is essential for heat recirculation in the conductive-tube configuration, still reduces low- $M$ performance. At higher values of $B$, thus lower $k_{w}$ (Fig. 10, upper), the wall temperature decreases to ambient on a scale smaller than the heat exchanger length whereas at lower $B$, thus higher $k_{w}$ (Fig. 10, lower), the entire wall is essentially isothermal at a temperature above ambient. In the latter case the system loses more heat to ambient than at higher $B$ without a corresponding benefit of increased heat recirculation, thus making the system more susceptible to extinguishment by heat losses. Of course if $B$ is too large, the temperature difference between the gas and the wall increases (because of the increased difficulty in transferring heat along the wall) which in turn decreases the amount of heat recirculation and leads to an upper limit on $B$.

Figure 11 shows the effect of $M$ on the minimum $\sqcap \tilde{T}$ supporting combustion for the conductive-tube configuration (analogous to Fig. 6 for the counter-current configuration). As with the counter-current configuration, without wall conduction $(B \square \infty)$ no small- $M$ extinction limit exists and for finite $B$, both small- $M$ and large- $M$ limits exist. In contrast to the countercurrent combustor, however, $B$ has a substantial effect on the high- $M$ extinction limit for the conductive-tube configuration for the reason discussed in the previous paragraph. The curves in Fig. 11 show a change in slope to a smaller value for small $M$ corresponding to the condition where heat losses are so severe that the entire heat exchanger is essentially at ambient temperature and no heat recirculation occurs. In this case the WSR operates with ambient inlet 
temperature $\left(\tilde{T}_{i}(1)=1\right)$ and the performance of the system can be described by Eq. (15) only. This behavior also occurs in a similar manner for the counter-current combustor but is not seen in Fig. 6 because it occurs at the same values of $\sqcap \tilde{T}$ as for the conductive-tube combustor, which is well off the scale of Fig. 6.

A comparison of Figs. 6 and 11 shows that the inherent performance of counter-current burners is superior to that of conductive-tube burners having the same reaction rate and heat loss parameters. In particular, when $B$ is considered to be a design parameter that can be optimized independently for the two configurations, for all $M$ the minimum $\sqcap \tilde{T}$ required to sustain combustion is lower for counter-current burners. For example, at $M=0.1$ the minimum $\sqcap \tilde{T}$ is about 1.25 for the counter-current burner and 2.15 for the conductive-tube burner and at $M=$ 0.01 the respective values are about 1.0 and 1.9. This difference is particularly noteworthy since the wall boundary condition at the WSR is perhaps the most favorable possible for combustor performance. The better performance of the counter-current burner is fundamentally due to the fact that there is less heat loss penalty for heat recirculation in this case. Specifically, as discussed in the counter-current analysis, wall thermal conduction leads to lower WSR temperatures when heat losses are present. In the conductive-tube case this wall conduction is the only mechanism for heat recirculation and thus must be present. In contrast, for the countercurrent case heat recirculation can be accomplished without wall thermal conduction, thereby more effectively decoupling heat loss from heat recirculation.

\section{Application to microscale devices}

Well-instrumented macroscale experiments are valuable tools for predicting microscale performance by invoking similitude (constant dimensionless parameters) since microscale devices are notoriously difficult to instrument. The analysis presented here may be useful for this purpose. For geometrically similar devices $\left(d \sim L \sim w \sim \square_{v}\right)$ with laminar flow $(h \sim 1 / d)$ it is easily shown that $M \sim U d / \square_{g}, B \sim k_{g} / k_{w}, D a \sim d^{2} Z / \square_{g}$ and $H=$ constant, where $\square_{g}$ is the gas thermal diffusivity. The biggest challenge is to maintain constant $M$ and $D a$ simultaneously as $d$ decreases. This would require (since $\square_{g} \sim P^{-1}$, where $P$ is the pressure) $P \sim d^{-2}$ and $U \sim d$. Changing pressure is problematic, however, since the overall reaction rate parameters $Z$ and $E$ are generally pressure-dependent. If pressure is fixed then geometrical similarity cannot be 
maintained. Similitude could be maintained with $U=$ constant, $L \sim d^{3}, w \sim d$ and $\square_{w} \sim d^{5}$, but because of manufacturing limitations this is not practical for large ranges of $d$. Perhaps the most viable scaling approach, is to employ geometrical similarity, constant pressure, $U \sim d^{-1}$ (thus constant $M$ and $R e$ ) and to maintain reaction rate similarity increase the fuel concentration $\sqcap \tilde{T}$ such that the right-hand side of Eq. (15) is constant even though $D a$ decreases with decreasing $d$. For example, for the counter-current combustor with fixed $M=0.01, B=10^{4}, H=0.05, \square=70$ and initial values $\sqcap \tilde{T}=1.1$ and $D a=10^{7}$, as $d$ is decreased from its nominal value $\left(d_{o}\right)$ the required $\sqcap \tilde{T}$ are well fit by the relation $\Pi \tilde{T}=1.07+0.03\left(d / d_{o}\right)^{-2}$. The temperature profiles are nearly identical for these values of $\sqcap \tilde{T}$. Note that according to this expression $\sqcap \tilde{T}$ rises rapidly as the scale $(d)$ decreases. This point is of particular interest for microscale combustion applications, where it may not be of special value to burn very lean mixtures or fuels with very low heating value but heat losses will be extremely problematic even for near-stoichiometric mixtures due to the enormous surface area to volume ratios associated with microscale devices.

\section{Discussion and conclusions}

A simple model of heat-recirculating burners was developed, including heat transfer from product to reactant streams in a counter-current heat exchanger, heat loss from both streams to ambient, thermal conduction along the dividing wall between the two streams and chemical reaction in a well-stirred reactor. The predicted effects of non-dimensional heat loss $(H)$ and fuel concentration $(\sqcap \tilde{T})$ are found to be straightforward. (The effects of Damköhler number $(D a)$ and activation energy $(\square)$, not shown in this paper, also follow the expected trends.) In contrast, the effects of mass flux $(M)$ and Biot number $(B)$ are neither straightforward nor even monotonic. In particular, extinction limits are predicted at both large $M$ (due to blow-off type limits well known for WSRs [21]) and small $M$ (due to heat losses which are not important for the large- $M$ limit). Most significantly, the small- $M$ extinction limit occurs only with wall conduction (finite $B$ ) because without this damping factor, as $M$ decreases the amount of heat recirculated (relative to heat generation) increases without bound. The importance of wall conduction cannot be overstressed since without wall conduction (infinite B), even with low reactivity fuels (low Da or $\sqcap \tilde{T})$ or large heat losses no small-M limit exists. This is because as $M$ decreases, heat recirculation increases (causing higher WSR temperatures and reaction rates) and WSR 
residence times increase; at sufficiently low $M$ this combination is always capable of overcoming losses. With wall conduction, some thermal energy is transferred away from the WSR region, re-deposited into the gas, then lost to ambient, which leads to small- $M$ limits. It is emphasized that wall conduction is not a heat loss mechanism, instead it re-distributes thermal energy within the device. In the current model for the counter-current exchanger only gas-phase thermal energy can be lost to ambient.

It should also be noted that in a spiral heat exchanger where (unlike the linear exchanger modeled here) the view factor of the dividing wall with itself is non-zero, the radiative heat transfer between walls would have a similar effect to streamwise wall conduction since this type of radiative transfer would also increase heat transfer within the solid phase without a corresponding increase in heat exchange with the gas. We have observed preliminary evidence of this in detailed numerical computations of Swiss Roll combustor performance [23] - the peak temperature in the combustor decreases when radiative transfer is included. Of course, if the gases were sufficiently absorbing, radiation could increase heat exchange with the gas and thus increase combustor temperatures, but the typical Planck mean absorption length of combustion products $(1-2 \mathrm{~m})$ far exceeds channel dimensions of laboratory-scale or microscale apparatuses and thus significant participation from gas-phase radiation would not be expected.

While streamwise wall conduction dominates burner performance, with the thermallythin wall model employed here spanwise conduction (across the wall) results in no temperature gradients and thus does not affect performance. This is considered realistic since for practical burner materials and dimensions, wall thermal resistance is much lower than gas-phase thermal resistance (see Counter-current combustor - Approach).

An alternative configuration of combustion stabilized at the exit of a tube, where heat recirculation occurs via conduction through tube wall was also analyzed. Although this configuration certainly qualifies as a heat-recirculating combustor, its performance was found to be inferior to that of the counter-current configuration in that much higher dimensionless fuel concentrations are required to sustain combustion at the same $M$.

It is believed that the conclusions of this study, while based on highly simplified transport and chemistry sub-models, are applicable to real devices also, particularly regarding effects of wall conduction. Similar trends would be expected with complex chemistry if the overall activation energy is large. Using constant overall heat transfer coefficients is reasonable for 
laminar flow, though for turbulent flow at high $\operatorname{Re}$, roughly $h_{1} \sim \dot{m}$, thus $M \approx$ constant (though the Damköhler number $D a$ is proportional to $h_{l}^{-1}$ and would decrease with increasing $\dot{m}$, thus leading to extinction limits at large $M$ even for turbulent flow.) Also, linear and spiral heat exchangers will have different $H$ but probably similar response to heat loss. The WSR combustion model is considered reasonable although approximate since all diffusive transport within the WSR is neglected. Moreover, catalytic combustion is advantageous for small- $M$ heat recirculating burners $[3,5]$. If catalyst is present only in the WSR volume, the WSR model is likely reasonable for catalytic combustion at small $M$ where residence times are long and reaction is kinetically limited (rather than potentially transport limited as at large $M$ ).

\section{Acknowledgements}

This work was supported by the DARPA Microsystems Technology Office, under contracts DABT63-99-C-0042 and N66001-01-108966.

\section{Nomenclature}

$A_{R} \quad$ WSR area (replaces WSR volume in three-dimensional problems)

$a \quad$ See Eqs. (5), (22)

$B \quad$ Scaled Biot number $=2 h_{1} L^{2} / k_{w} \square$

$b \quad$ See Eqs. (5), (22)

c Integration constants (Eq. 5)

$C_{p} \quad$ Heat capacity at constant pressure

$D a \quad$ Damköhler number $=\square_{g} C_{P} A_{R} Z / L h_{1}$

$d \quad$ Channel height

$d_{H} \quad$ Hydraulic diameter

E Activation energy

$H \quad$ Dimensionless heat loss coefficient $=h_{2} / h_{1}$

$g \quad$ See Eq. (22)

$h_{1} \quad$ Heat transfer coefficient to divider wall

$h_{2} \quad$ Heat loss coefficient to ambient

$k \quad$ Thermal conductivity

$L \quad$ Heat exchanger length

$M \quad$ Dimensionless mass flux $=\dot{m} C_{P} / h_{l} L$

$\dot{m} \quad$ Mass flow rate per unit depth

NTU Number of Transfer Units

$\mathrm{Nu} \quad$ Nusselt number $=\mathrm{h}_{1} \mathrm{~d} / \mathrm{k}_{\mathrm{g}}$

Pr Prandtl number $\equiv \square_{g} C_{P} / k_{g}$

$R \quad$ Gas constant

Re Reynolds number $=\dot{m} \mathrm{~d} / \mu$

$T$ Temperature 
$\tilde{T} \quad$ Dimensionless temperature $=T / T_{\infty}$

$U$ bulk flow velocity

WSR Well-stirred reactor

w Channel depth

$\mathrm{x} \quad$ Streamwise coordinate

$\tilde{x} \quad$ Dimensionless streamwise coordinate $=x / L$

$Z \quad$ Pre-exponential factor in reaction rate expression

( Non-dimensional activation energy (Zeldovich number) $=E / R T_{\infty}$

$\sqcap \tilde{T} \quad$ Temperature rise for adiabatic complete combustion

$\square \quad$ Heat exchanger effectiveness

$\mu \quad$ Dynamic viscosity

$\square \quad$ density

$\square \quad$ Dividing wall thickness

Subscripts

e product side of heat exchanger

g gas

i reactant side of heat exchanger

w dividing wall

$\infty \quad$ ambient conditions

\section{References}

1. S. A. Lloyd, F. J. Weinberg, Nature 251 (1974) 47-49.

2. S. A. Lloyd, F. J. Weinberg, Nature 257 (1975) 367-370.

3. L. Sitzki, K. Borer, S. Wussow, E. Schuster, P. D. Ronney, A. Cohen, AIAA Paper 2001-1087 (2001).

4. F. J. Weinberg, D. M. Rowe, G. Min, P. D. Ronney, Proc. Combust. Inst. 29 (2002) 941-947.

5. J. Vican, B. F. Gajdeczko, F. L. Dryer, D. L. Milius, I. A. Aksay, Proc. Combust. Inst. 29 (2002) 909-916.

6. A. C. Fernandez-Pello, Proc. Combust. Inst. 29 (2002) 883-899.

7. A. R. Jones, S. A. Lloyd, F. J. Weinberg, Proc. Roy. Soc. Lond. A. 360 (1978) 97-115.

8. P. D. Ronney, Proc. Combust. Inst. 27 (1998) 2485-2506.

9. B. Cooley, D. Walther, A. C. Fernandez-Pello, "Exploring the Limits of Microscale Combustion," 1999 Fall Technical Meeting, Western States Section/Combustion Institute, Irvine, CA, October 25-26, 1999. 
10. R. R. Peterson, J. M. Hatfield, "A Catalytically Sustained Microcombustor Burning Propane," Proc. 2001 International Mechanical Engineering Congress and Exposition (IMECE), New York, November 11-16, 2001.

11. J. A. Wünning, J. G. Wünning, Prog. Energy Combust. Sci. 23 (1997) 81-94.

12. M. Katsui, T. Hasegawa, Proc. Combust. Inst. 27 (1998) 3135-3146.

13. K. Maruta, K. Muso, K. Takeda, T. Niioka, Proc. Combust. Inst. 28 (2000) 2117-2123.

14. P. G. Kroeger, in: Advances in Cryogenic Engineering, K. D. Timmerhaus (Ed.), Plenum Press, New York, 1967, pp. 363-372

15. G. Venkatarathnam, S. P. Narayanan, Cryogenics 39 (1999) 811-819.

16. X. Yin, H. H. Bau, J. Heat Trans. 188 (1996) 482-485.

17. R. B. Peterson, Microscale Thermophysical Engineering 3 (1999) 17-30.

18. Y. B. Zel'dovich, Combust. Flame 39 (1980) 219.

19. R. K. Shah, A. L. London, Laminar Flow: Forced Convection in Ducts, Academic Press, 1978.

20. J. N. deRis, Twelfth Symposium (International) on Combustion, Combustion Institute, 1969, p. 241.

21. I. Glassman, Combustion ( ${ }^{\text {rd }}$ Ed.), Academic Press, 1996.

22. K. Maruta, K. Takeda, J. Ahn, K. Borer, L. Sitzki, P. D. Ronney, O. Deutchman, Proc. Combust. Inst. 29 (2002) 957-963.

23. J. Kuo, C. Eastwood, L. Sitzki, K. Borer, P. D. Ronney, "Numerical modeling of heat recirculating burners," $3^{\text {rd }}$ Joint US Sections Meeting, Combustion Institute, Chicago, IL, March 2003. 


\section{Figure Captions}

Figure 1. Schematic diagram of heat exchanger / combustor configurations analyzed. Upper: counter-current system; lower; conductive tube.

Figure 2. Temperature profiles in the counter-current heat exchanger for $D a=\infty, M=0.2, \sqcap \tilde{T}=$ 1.5. Top: $H=0, B=\infty$; middle: $H=1, B=\infty$; bottom: $H=0, B=10$.

Figure 3. Effect of mass flux on WSR temperature in the counter-current combustor for infinite reaction rates $(D a \square \infty)$ with $\sqcap \tilde{T}=1.5$.

Figure 4. Effect of mass flux on WSR temperature in the counter-current combustor for finite reaction rates $\left(D a=10^{7}\right)$ with $\sqcap \tilde{T}=1.5$. For reference, the adiabatic, conduction-free infiniterate curve from Fig. 3 is also shown.

Figure 5. Effect of Biot number $(B)$ on WSR temperature in the counter-current combustor for finite reaction rates $\left(D a=10^{7}\right)$ with $H=1.5$ and $\sqcap \tilde{T}=1.5$, for several fixed values of the mass flux $M$.

Figure 6. Effect of mass flux on fuel concentration (expressed as $\sqcap \tilde{T}$ ) at the extinction limit in the counter-current combustor for varying values of the Biot number $(B) . D a=10^{7}, H=0.05$.

Figure 7. Effect of mass flux on Biot number $(B)$ at the extinction limit in the counter-current combustor for varying values of the heat loss coefficient $(H) . D a=10^{7}, \sqcap \tilde{T}=1.5$.

Figure 8. Effect of the boundary condition on the heat exchanger dividing wall on the predicted extinction limits of the counter-current combustor. (a): Comparison of convection boundary conditions (for $B^{*}=1$ ) to adiabatic walls in Biot number $(B)$ - mass flux $(M)$ space. (b): same results plotted in terms of limit mass flux $(M)$ as a function of wall thickness $(\square 2 L)$.

Figure 9. Effect of mass flux on WSR temperature in the conductive-tube combustor for finite reaction rates $\left(D a=10^{7}, \square=70\right)$ with $\sqcap \tilde{T}=2.25$. Also shown for reference is the case for 
infinite reaction rate $D a=\infty$ without heat loss $(H=0)$ or wall conduction $(B=\infty)$. For the finite$D a$ case without wall conduction the values of mass flux $M$ have been multiplied by 1000 .

Figure 10. Temperature profiles in the conductive-tube combustor for $D a=10^{7}, M=0.06, \sqcap \tilde{T}=$ 2.25, $H=0.05$. Top: $B=10$; bottom: $H=0, B=100$.

Figure 11. Effect of mass flux on fuel concentration (expressed as the temperature rise for adiabatic complete combustion, $\sqcap \tilde{T}$ ) at the extinction limit in the conductive-tube combustor for varying values of the Biot number $(B) . D a=10^{7}, H=0.05$. 

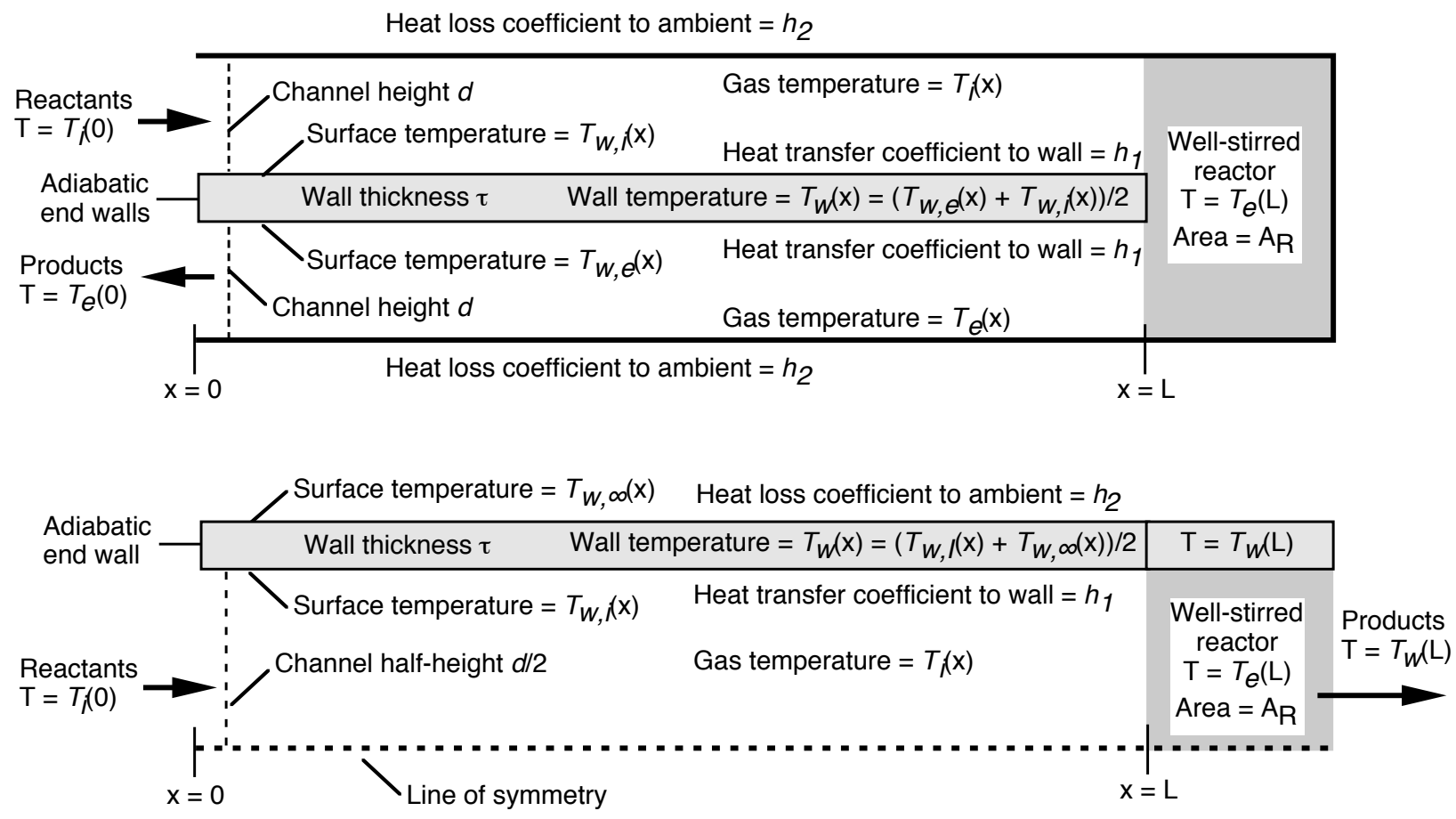

Figure 1. Schematic diagram of heat exchanger / combustor configurations analyzed. Upper: counter-current system; lower; conductive tube. 


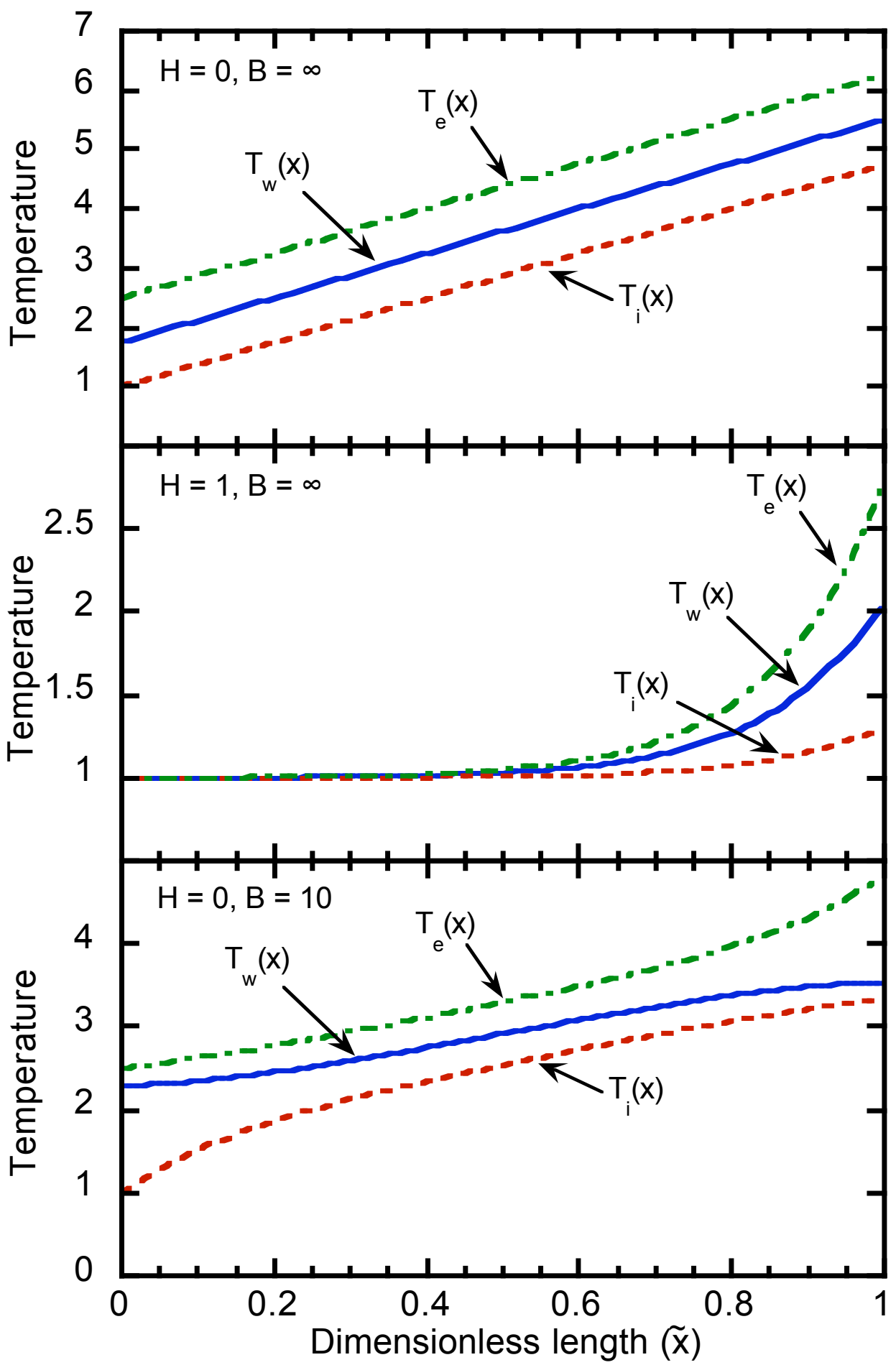

Figure 2. Temperature profiles in the counter-current heat exchanger for $D a=\infty, M=0.2, \sqcap \tilde{T}=$ 1.5. Top: $H=0, B=\infty$; middle: $H=1, B=\infty$; bottom: $H=0, B=10$. 


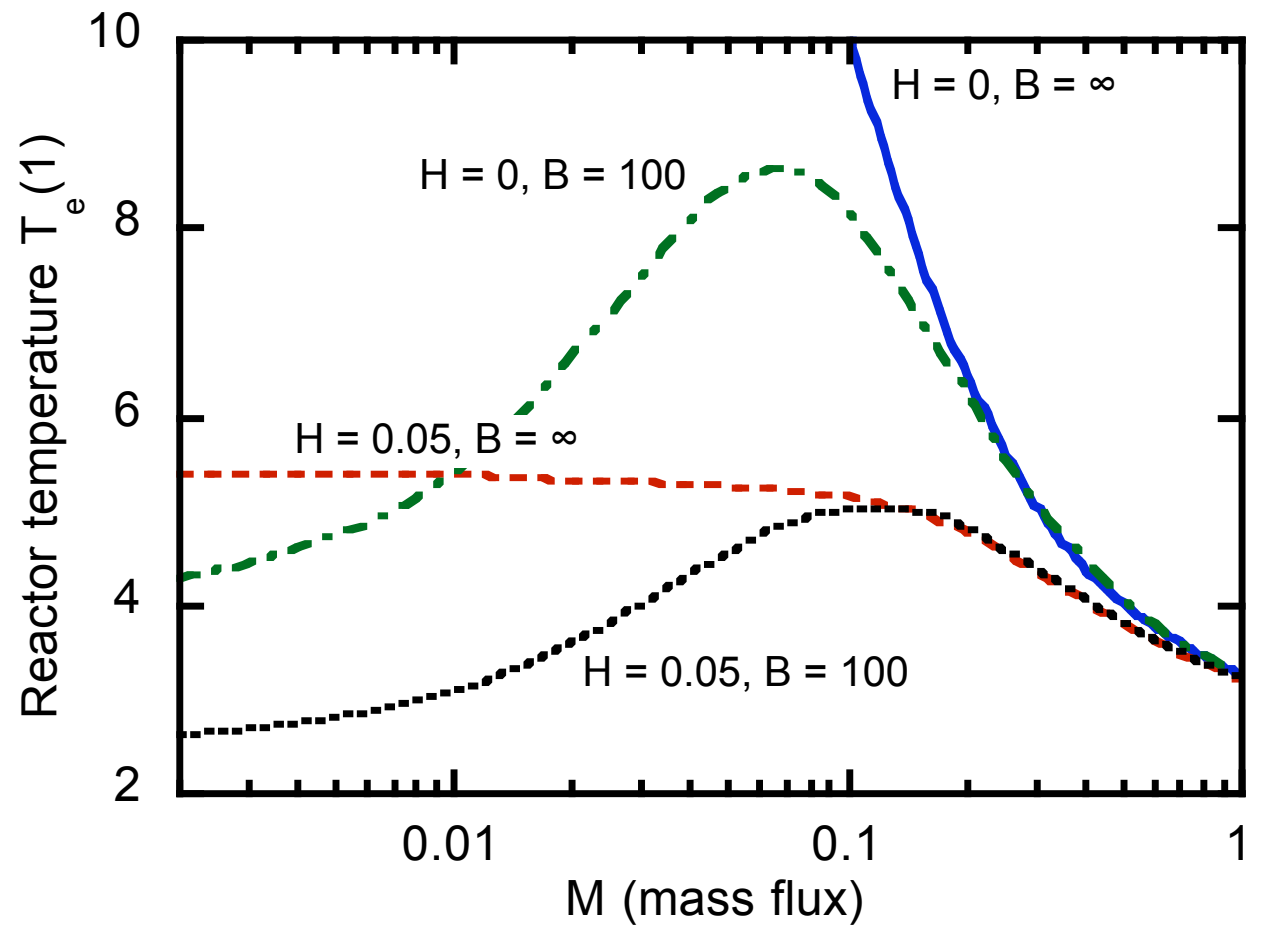

Figure 3. Effect of mass flux on WSR temperature in the counter-current combustor for infinite reaction rates $(D a \square \infty)$ with $\Pi \tilde{T}=1.5$. 


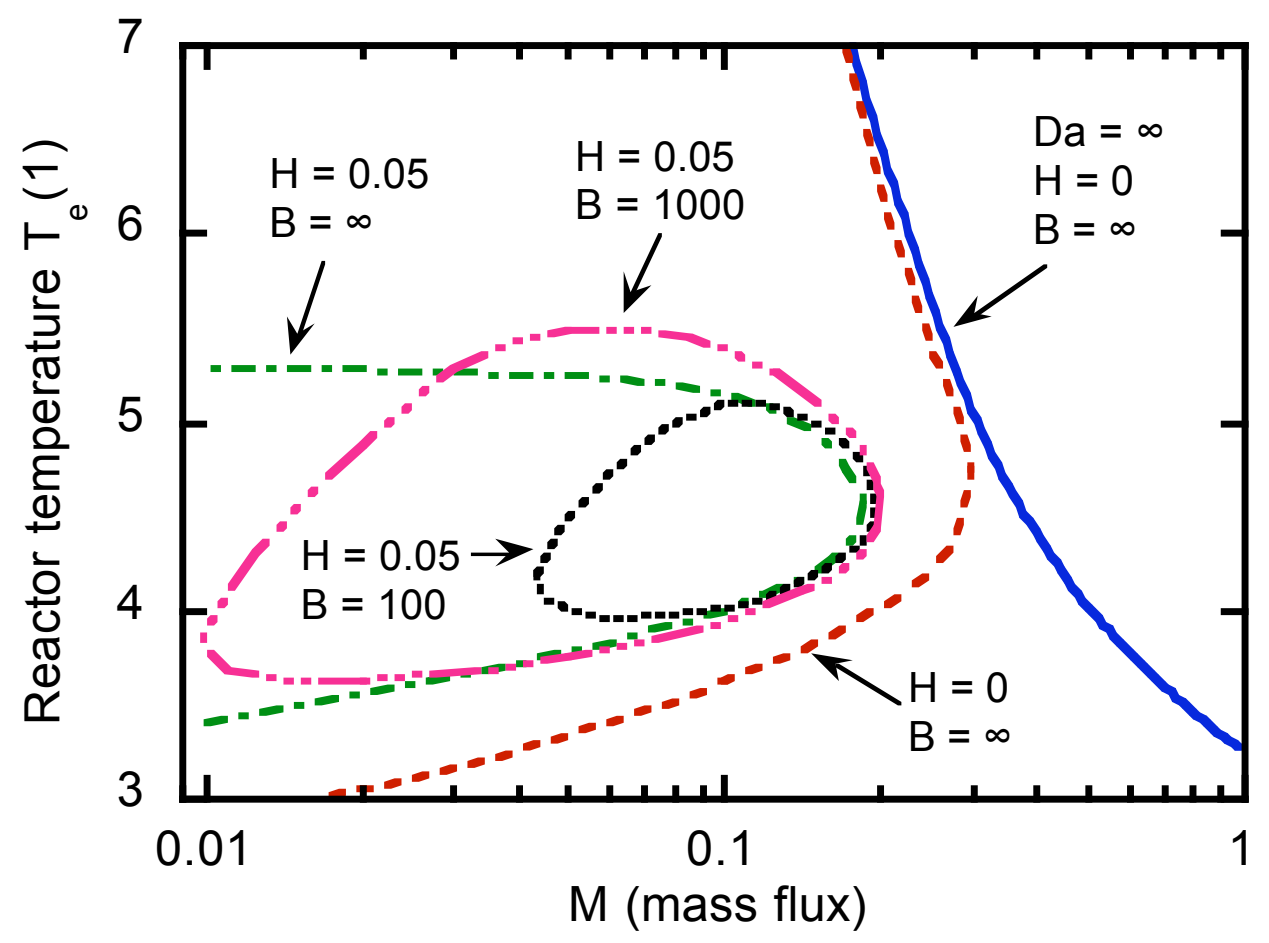

Figure 4. Effect of mass flux on WSR temperature in the counter-current combustor for finite reaction rates $\left(D a=10^{7}\right)$ with $\sqcap \tilde{T}=1.5$. For reference, the adiabatic, conduction-free infiniterate curve from Fig. 3 is also shown. 


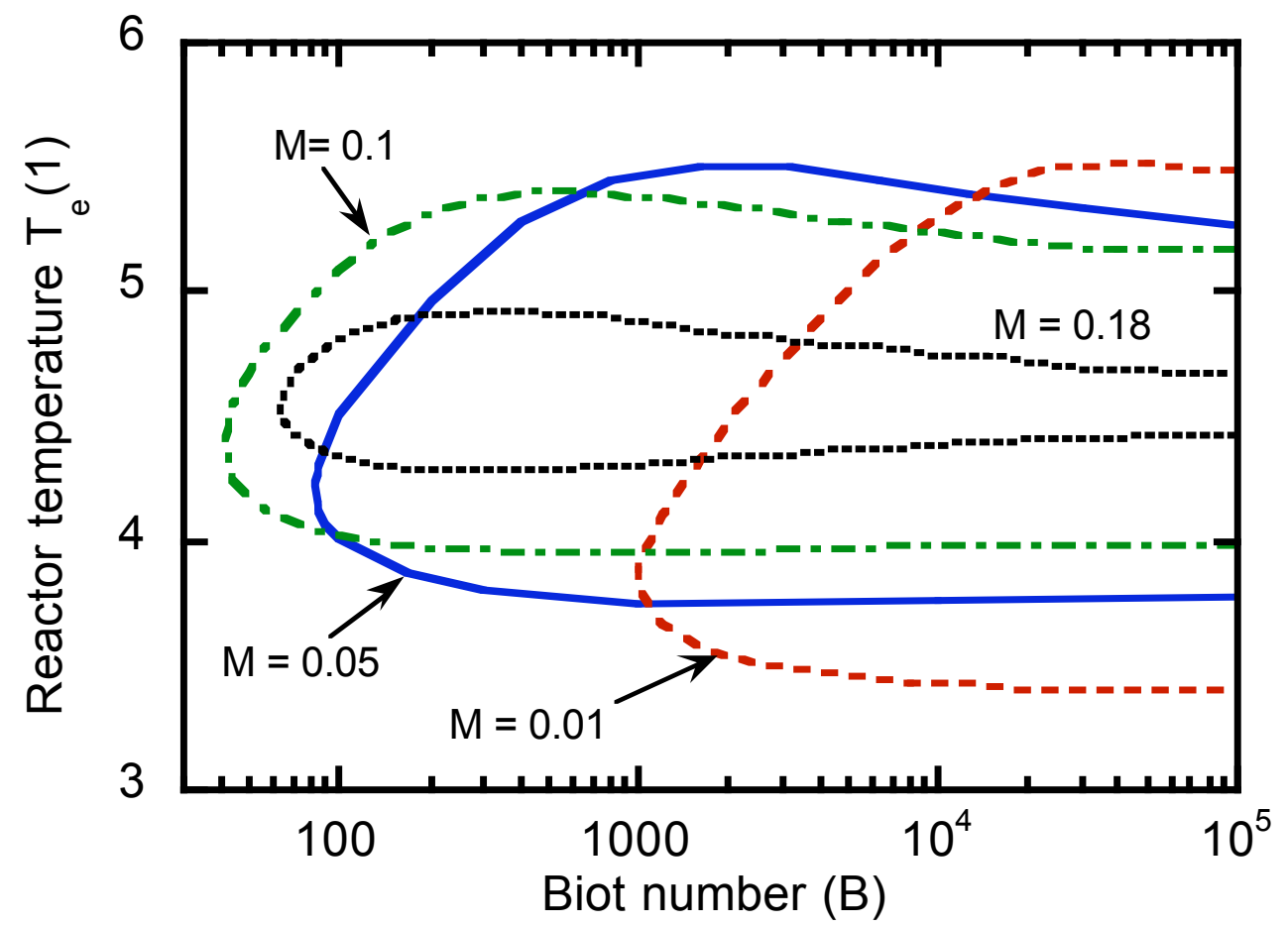

Figure 5. Effect of Biot number $(B)$ on WSR temperature in the counter-current combustor for finite reaction rates $\left(D a=10^{7}\right)$ with $H=1.5$ and $\sqcap \tilde{T}=1.5$, for several fixed values of the mass flux $M$. 


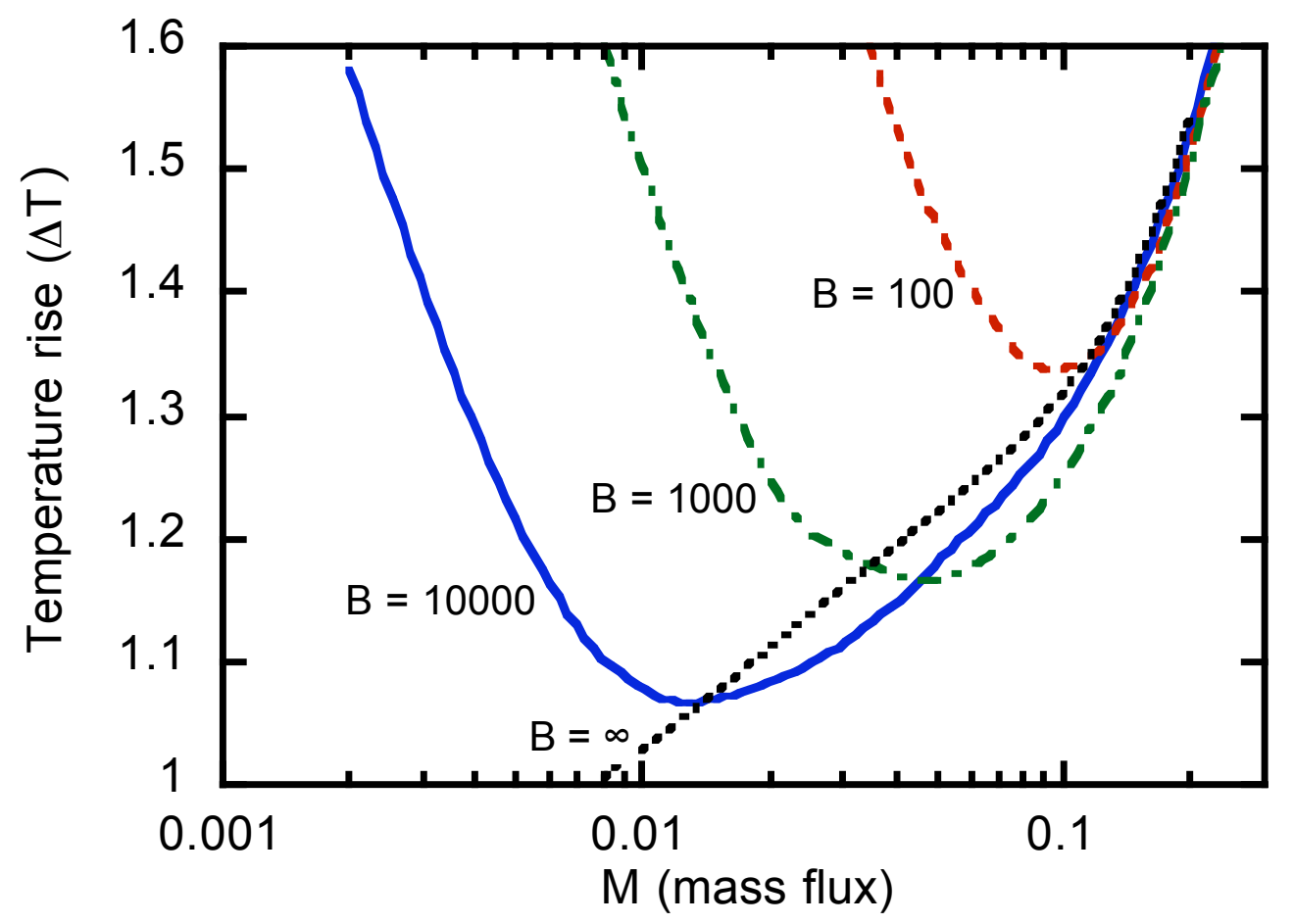

Figure 6. Effect of mass flux on fuel concentration (expressed as the temperature rise for adiabatic complete combustion, $\Pi \tilde{T}$ ) at the extinction limit in the counter-current combustor for varying values of the Biot number $(B) . D a=10^{7}, H=0.05$. 


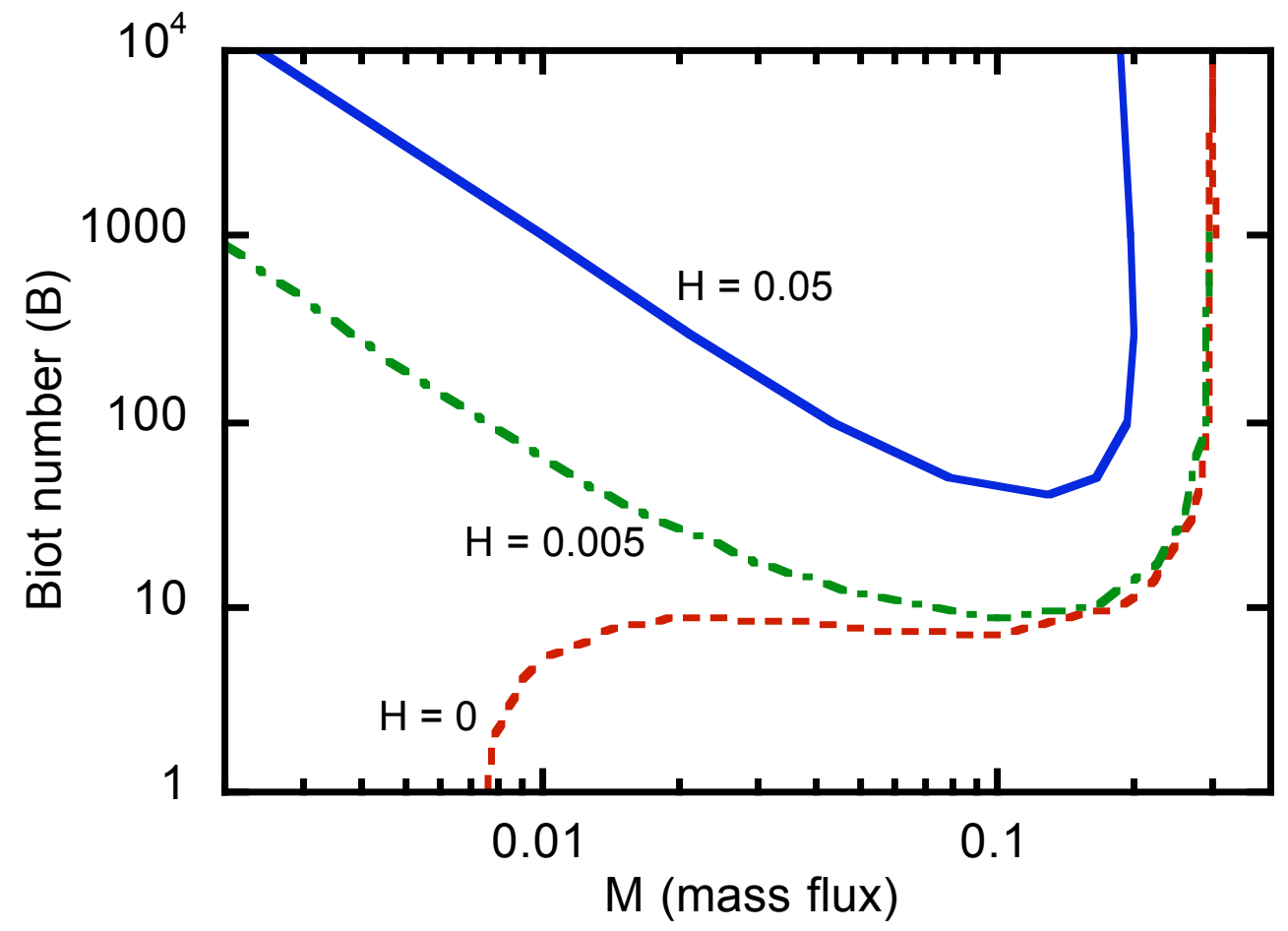

Figure 7. Effect of mass flux on Biot number $(B)$ at the extinction limit in the counter-current combustor for varying values of the heat loss coefficient $(H) . D a=10^{7}, \Pi \tilde{T}=1.5$. 

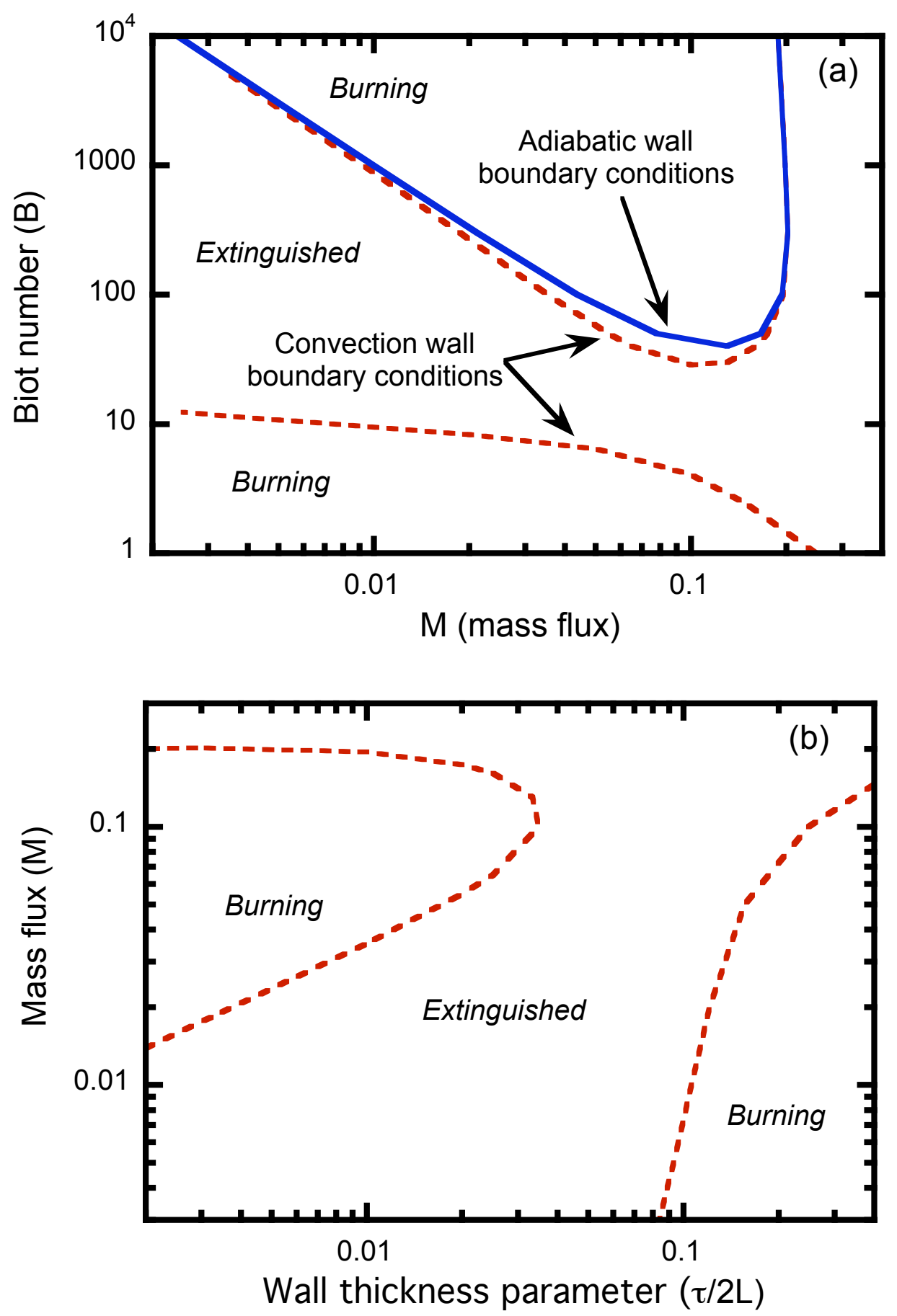

Figure 8. Effect of the boundary condition on the heat exchanger dividing wall on the predicted extinction limits of the counter-current combustor. (a): Comparison of convection boundary conditions (for $B^{*}=1$ ) to adiabatic walls in Biot number $(B)$ - mass flux $(M)$ space. (b): same results plotted in terms of limit mass flux $(M)$ as a function of wall thickness $(\square 2 L)$. 


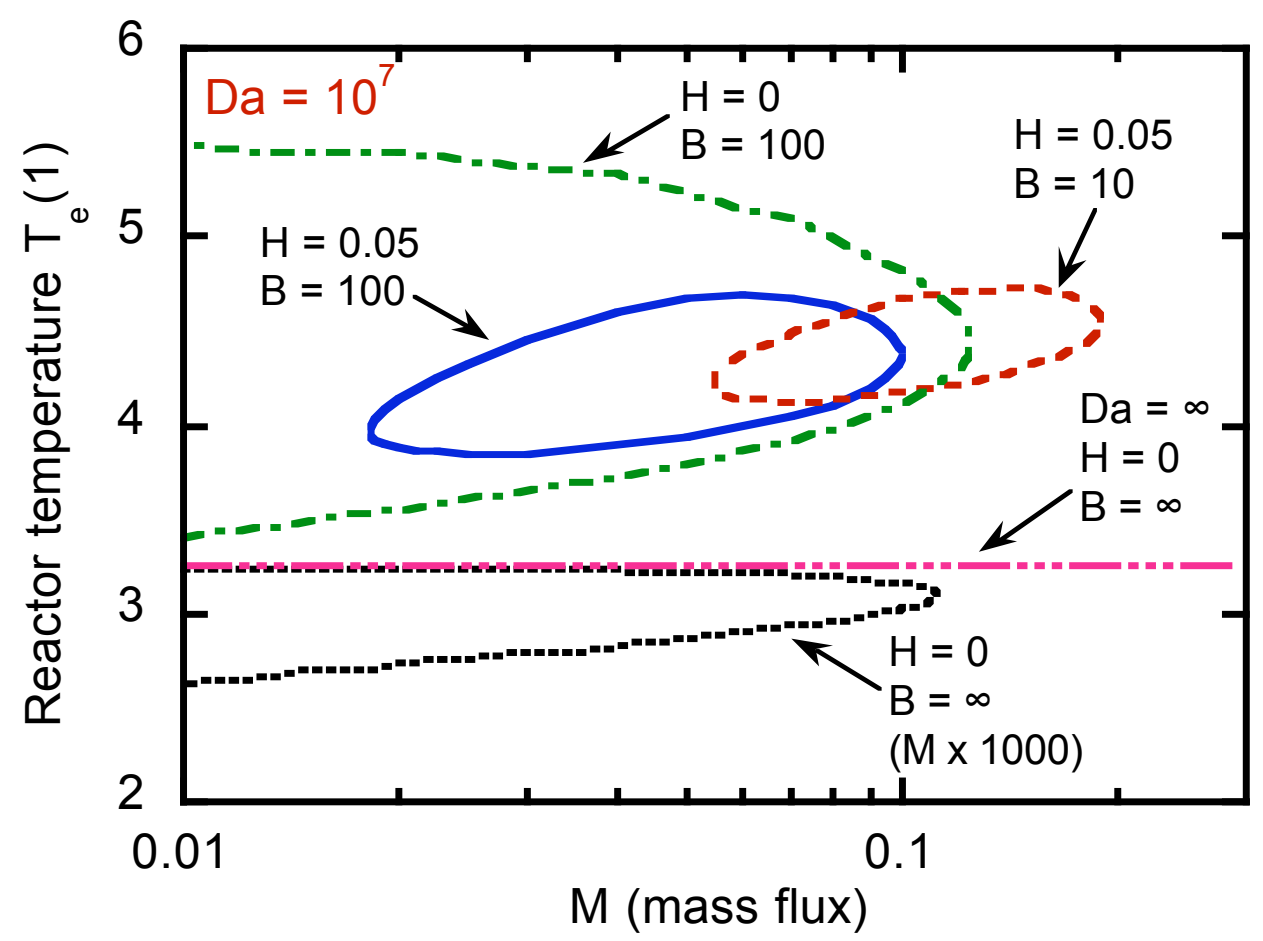

Figure 9. Effect of mass flux on WSR temperature in the conductive-tube combustor for finite reaction rates $\left(D a=10^{7}, \square=70\right)$ with $\sqcap \tilde{T}=2.25$. Also shown for reference is the case for infinite reaction rate $D a=\infty$ without heat loss $(H=0)$ or wall conduction $(B=\infty)$. For the finite$D a$ case without wall conduction or heat loss the values of mass flux $M$ have been multiplied by 1000. 


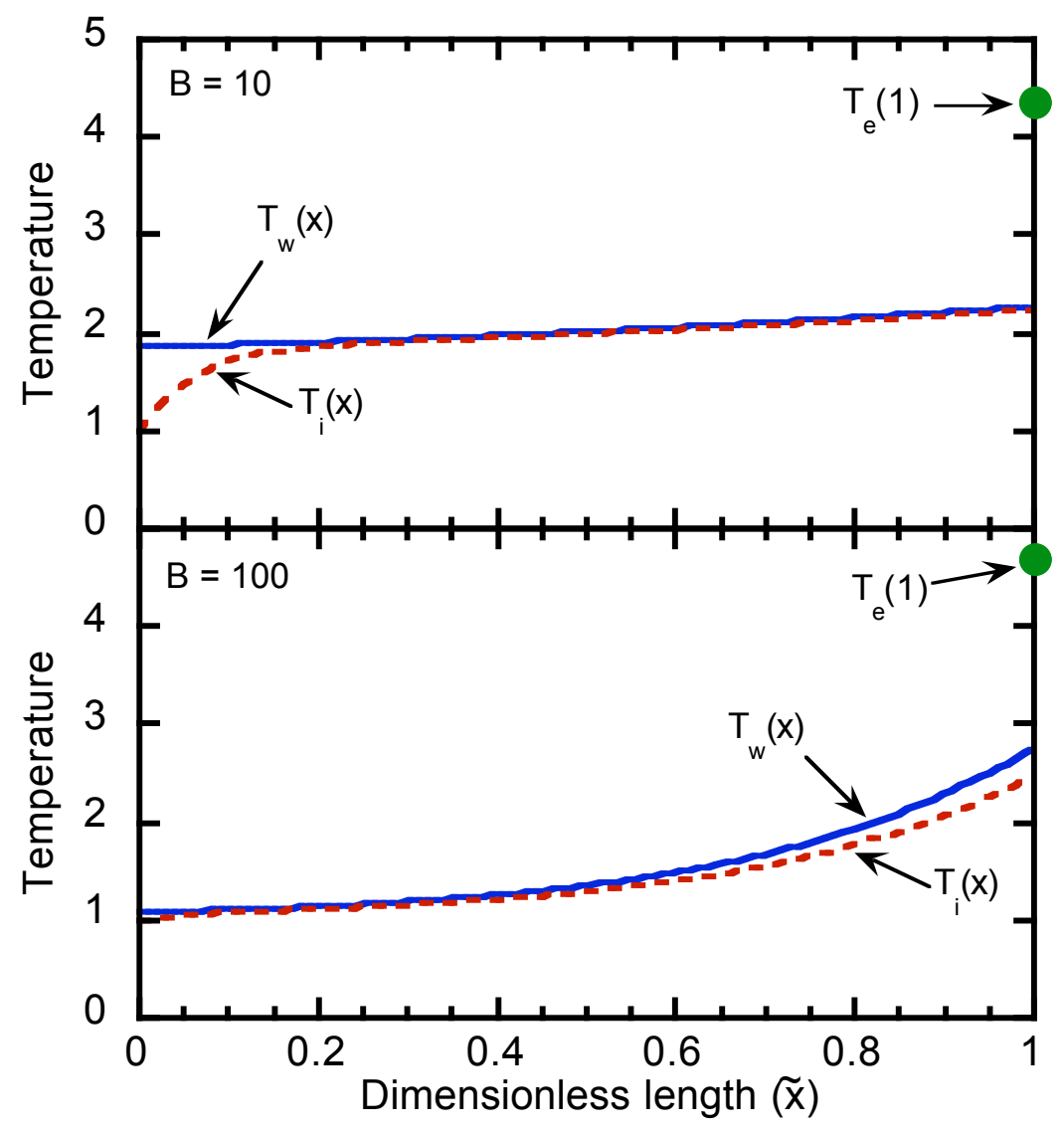

Figure 10. Temperature profiles in the conductive-tube combustor for $D a=10^{7}, M=0.06, \Pi \tilde{T}=$ 2.25, $H=0.05$. Top: $B=10$; bottom: $H=0, B=100$. 


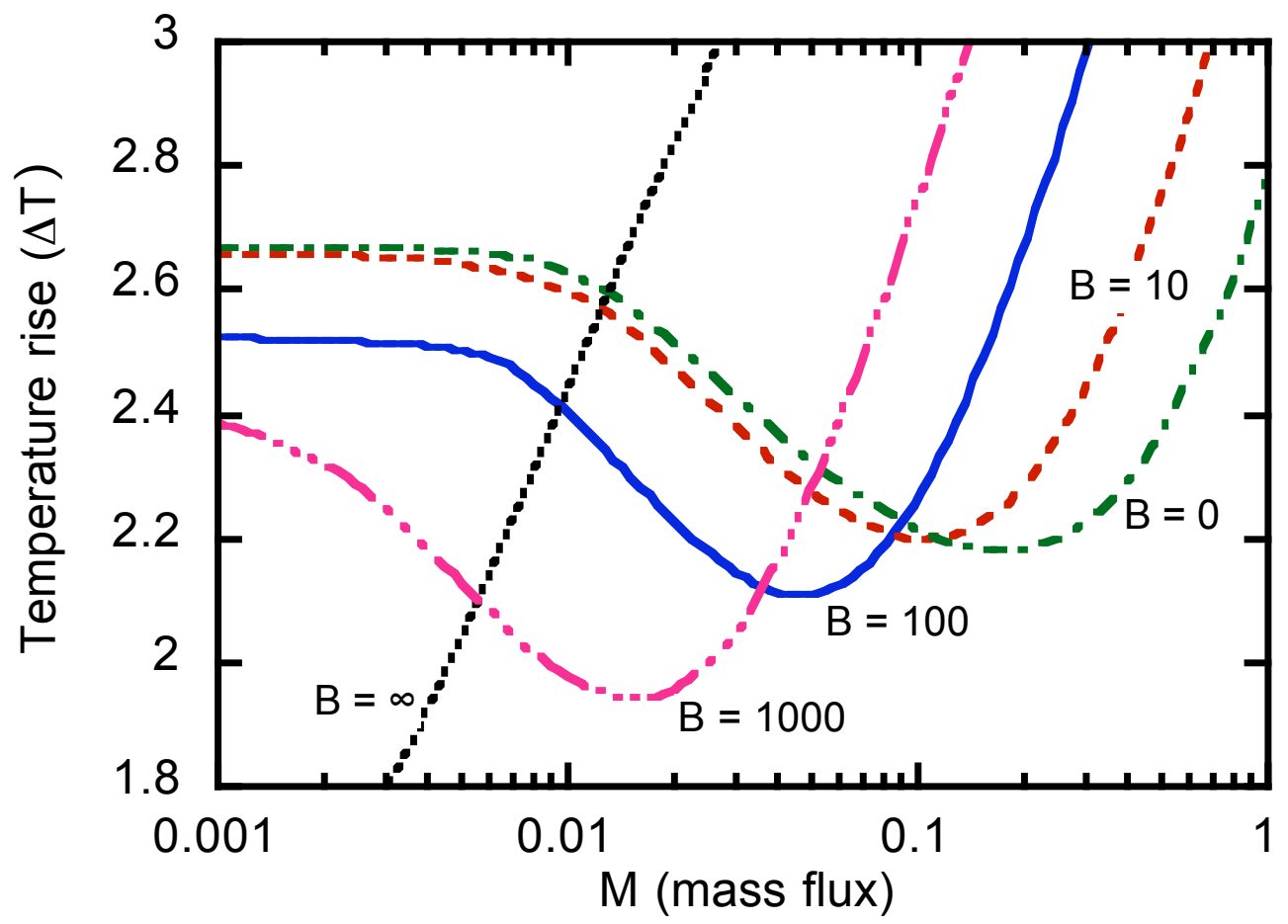

Figure 11. Effect of mass flux on fuel concentration (expressed as the temperature rise for adiabatic complete combustion, $\sqcap \tilde{T}$ ) at the extinction limit in the conductive-tube combustor for varying values of the Biot number $(B) . D a=10^{7}, H=0.05$. 